\title{
How Many Species Can Two Essential Resources Support?
}

\author{
Bingtuan $\mathrm{Li}^{*}$ \\ Department of Mathematics \\ University of Utah \\ Salt Lake City, Utah 84112 \\ Hal Smith ${ }^{\dagger}$ \\ Institute for Mathematics and Its Applications \\ University of Minnesota \\ 400 Lind Hall \\ 207 Church Street S.E. \\ Minneapolis, Minnesota 55455
}

\begin{abstract}
A chemostat model of $n$ species of microorganisms competing for two perfectly complementary, growth-limiting nutrients is considered. Sufficient conditions are given for there to be a single winning species and for two species to coexist, driving the others to extinction. In case $n=3$, it is shown that every solution converges to one of the single-species or two-species steady states, and hence the dynamics of the model is completely determined. The results generalize those of Hsu, Cheng, and Hubbell [15] as well as Butler and Wolkowicz [6] who considered two species.
\end{abstract}

Short Title. competition for two resources

Keywords. chemostat, competition for two resources, competitive exclusion principle, coexistence, global asymptotic behavior, competitive system

AMS Classification. 92A15, 92A17, 34C15, 34C35

* Part of this work was done when this author was a postdoctoral associate at the Institute for Mathematics and its Applications (IMA), University of Minnesota. The author thanks the IMA for its support with funds provided by the National Science Foundation.

${ }^{\dagger}$ This author thanks the IMA for support as a visiting fellow; also supported by NSF Grant DMS 9700910 


\section{Introduction}

More generally, given $k$ essential resources, how many species can be supported in purely exploitative competition for the given resources in a spatially and temporally homogeneous environment? It has long been known [1] that generically speaking, equilibrium coexistence is impossible if $n>k$ and, as competition rarely generates oscillations, one might expect that not more than $k$ species can be supported by $k$ (non-reproducing) resources. Certainly, if $n>k$ the $n$ species cannot be uniformly persistent in the usual sense of that term [37] for this would imply a coexistence equilibrium [42] which does not exist. This observation can also be found in [1]. However, numerical simulations in a recent paper of Huisman and Weissing [17] strongly suggest that up to six species can be supported by three resources and as many as twelve species can be supported on five resources. Their simulations show that certain solutions of the standard model of $k$ species with $k$ resources oscillate (periodically if $k=3$ and chaotically if $k=5$ ) and that these oscillatory communities (solutions) can be successively invaded by one species after another up to the total numbers given above. These results have important implications for the planktonic paradox-Why can so many plankton species seemingly coexist on so few limiting resources? (see also Sommer [29]). One does not need to posit exogenous factors such as seasonal or diurnal forcing.

Fairly complete and mathematically rigorous work on this problem has been limited to two cases: $k=1$ and arbitrary $n$ and $k=n=2$. We review these below. Our focus here is on the case $k=2$ and $n>2$, and to end the suspense, we are unable to answer the question in the title for general $n$. Criteria for existence of heteroclinic cycles of steady states as well as a particular example for such existence are given for the case $n=4$. The significance of such a cycle is two-fold. First, it is a potential limit set for the dynamics of the system (although apparently not for 
the example mentioned) or for a larger system containing the given one. Secondly, it is possible to envision the bifurcation of a very long-period periodic orbit from such a cycle. For $n=3$ we show that at least one species becomes extinct. Every solution approaches a single-species or two-species steady state. However, as already noted for the case $n=k=2$ different initial data may lead to different winners and losers. Cases where as many as three asymptotically stable equilibria are possible. Even for $n=3$ there are a large number of possible phase diagrams and we give a fairly complete picture of these. For general $n$, we give a number of results which imply that one or another of the (one or two-population) steady states is globally attractive for positive initial data and we note situations where extinctions of certain species are inevitable.

The classical theory of ecological competition is attributed to Lotka [19] and Volterra [38]. It seeks to describe population dynamics without being specific about which resources are limiting and how effectively these limiting resources are utilized by competitors. These models are usually more phenomenological than predictive, as it is difficult to measure the important parameters in the theory without actually growing the species together in competition.

Over the past fifty years or so, a more mechanistic, resource-based theory of ecological competition has been developed (see, e.g, [12, 18, 24, 27, 34, 35]). This theory considers the dynamics of the resources explicitly as well as the population dynamics of the competing species. In comparison with the classical models, the resource-based models may be less general and more difficult to analyze ( see, e,g., $[1,16,39])$. However, the resource-based models are often more predictive because the parameters can be measured on species grown alone [9].

The simplest form of resource-based competition occurs in laboratory apparatus, called a chemostat or continuous culture. See the monograph of [31] for a description 
of chemostat in detail and for the general theory of the chemostat; other good sources are [8] and [36]. When a species grows on a single limiting nutrient, there is a so-called "break-even" concentration of this nutrient at which growth rate just balances removal rate. If there are more than one species competing for the nutrient and the functions for nutrient uptake are monotone increasing or non-monotone but with certain restrictions, then only the species with lowest break-even concentration survives $[5,14,20,21,40,41]$. This is often referred as the "competitive exclusion principle".

When there are two or more limiting nutrients in the chemostat, it becomes necessary to consider how the nutrients, once consumed, interact to promote growth. Leon and Tumpson [18] and Rapport [26] classify nutrients as perfectly complementary, perfectly substitutable, or imperfectly substitutable. Perfectly complementary nutrients are sources of different essential substances which are independently required for growth, such as a carbon source and a nitrogen source for a bacterium. The more modern term for complementary resources appears to be "essential resources" [8] as in the title. On the other hand, perfectly substitutable nutrients are alternative sources of an essential substance, and represent interdependent requirement for growth, such as two carbon sources for or two sources of phosphorous. The intermediate case is called imperfectly substitutable.

Chemostat models with two perfectly complementary nutrients have been studied in $[2,6,15,18,22,26,28,30,39]$ and related experiments are described in $[2,28,30]$. For models with two perfectly substitutable nutrients and two species, see [3, 4, 39]. For other related work, see $[2,7,25,36]$. In the case in which there are two perfectly complementary nutrients and two species and each competitor's functional response is monotone increasing, Leon and Tumpson [18] find conditions for the existence of a locally asymptotically stable interior steady state and hence conditions for 
the coexistence of two competitors. Hsu, Cheng, and Hubbell [15], on the other hand, give a complete global analysis of the model with two competitors under the assumption that consumption of the nutrients follows Michaelis-Menten kinetics, generalized to the two nutrient situation. They give biological conditions based on parameters that predict the outcome of the competition. They conclude that "each of the four outcomes of classical Lotka-Volterra two-species competition theory has multiple mechanistic origins in terms of consumer resource interactions." Their proofs are actually also valid for monotone increasing nutrient uptake functions. Butler and Wolkowicz [6] give different proofs in the case of monotone increasing response functions and two species. They also study the case in which at least one of the two competitors is inhibited by high concentration of the substrate. They construct examples for which there is a stable periodic solution and examples for which coexistence is possible but surprisingly neither competitor can survive in the absence of its rival.

In this paper, we consider the classical competition model which describes $n$ species competing exploitatively for two perfectly complementary nutrients $S$ and $R$. This paper is organized as follows. In the next section, we present the classical model (see [8]). Section 3 deals with existence and local stability of steady states, as well as a graphical interpretation of the stability conditions. Our main results are presented in section 4 and a discussion of their mathematical and biological significance is given in section 5. Proofs are deferred to a final section.

\section{The Model}

The classical model of $n$ species of microorganisms competing for two essential nutrients in a chemostat leads to the differential equations $[8,36]$ : 


$$
\begin{aligned}
& S^{\prime}(t)=\left(S^{0}-S(t)\right) D-\sum_{i=1}^{n} \frac{x_{i}(t)}{y_{S_{i}}} f_{i}(S(t), R(t)), \\
& R^{\prime}(t)=\left(R^{0}-R(t)\right) D-\sum_{i=1}^{n} \frac{x_{i}(t)}{y_{R_{i}}} f_{i}(S(t), R(t)), \\
& x_{i}^{\prime}(t)=x_{i}(t)\left(f_{i}(S(t), R(t))-D\right), \quad i=1,2, \ldots, n,
\end{aligned}
$$

where $S(0) \geq 0, R(0) \geq 0$, and $x_{i}(0)>0, i=1,2, \ldots, n$, and

$$
\left.f_{i}(S, R)=\min \left(p_{i}(S)\right), q_{i}(R)\right), \quad i=1,2, \ldots, n
$$

$S(t)$ and $R(t)$ denote the concentrations of the two essential nutrients at time $t$, and $x_{i}(t)$ denotes the density of the $i$ th population of microorganisms at time $t ; p_{i}(S)$ $\left.\left(q_{i}(R)\right)\right)$ represents the per-capita growth rate of the $i$ th population when nutrient $S$ is limiting (nutrient $R$ is limiting), and so $f_{i}(S, R)$ represents the rate of conversion of nutrient to biomass for the $i$ th population. We take the minimum here because the resources are perfectly complementary. We assume that growth rates adjust instantaneously to change in the nutrient concentration. $f_{i}(S, R) / y_{S_{i}}\left(f_{i}(S, R) / y_{R_{i}}\right)$ represents the uptake rate of nutrient $S$ (nutrient $R$ ) of the $i$ th population; and so $y_{S_{i}}$ and $y_{R_{i}}$ are growth yield constants. If only one feed bottle is used in the chemostat, then $S^{0}$ and $R^{0}$ denote, the concentrations of the growth limiting nutrients $S$ and $R$ respectively in the feed bottle, and $D$ denotes the flow rate of the chemostat. Here we assume that the growth vessel is well stirred, so that nutrients and microorganisms are removed in proportion to their concentrations. We also assume that individual death rates of populations are insignificant compared to the flow rate $D$.

If two separate feed bottles are used, with each containing only one nutrient, and input from each feed bottle to the growth vessel at different rates, say rate $D_{S}$ from the bottle containing nutrient $S$, and rate $D_{R}$ from the bottle containing nutrient $R$, then $D=D_{S}+D_{R}, S^{0}=\left(\bar{S}^{0} D_{S}\right) /\left(D_{S}+D_{R}\right)$, and $R^{0}=\left(\bar{R}^{0} D_{R}\right) /\left(D_{S}+D_{R}\right)$, where $\bar{S}^{0}$ and $\bar{R}^{0}$ represent the concentrations of nutrients $S$ and $R$, respectively, in each separate bottle. 
The functions $p_{i}: \mathbb{R}_{+} \rightarrow \mathbb{R}_{+}$and $q_{i}: \mathbb{R}_{+} \rightarrow \mathbb{R}_{+}$are assumed to be continuously differentiable with $p_{i}(0)=q_{i}(0)=0$ and satisfy:

$$
p_{i}^{\prime}(S)>0, S>0 \quad q_{i}^{\prime}(R)>0, R>0 .
$$

Furthermore, we assume that there exist positive extended real numbers $\lambda_{S_{i}}$ and $\lambda_{R_{i}}$ such that

$$
\begin{array}{ll}
p_{i}(S)<D & \text { for } S \in\left(0, \lambda_{S_{i}}\right) \quad \text { and } \quad p_{i}(S)>D \quad \text { for } S \in\left(\lambda_{S_{i}},+\infty\right) \\
q_{i}(R)<D & \text { for } R \in\left(0, \lambda_{R_{i}}\right) \quad \text { and } \quad q_{i}(R)>D \text { for } R \in\left(\lambda_{R_{i}},+\infty\right) .
\end{array}
$$

Thus $\lambda_{S_{i}}$ and $\lambda_{R_{i}}$ are the break-even concentrations for nutrient $S$ and $R$ respectively, when that nutrient is limiting. In the case where $p_{i}(S)<D\left(q_{i}(R)<D\right)$ for all $S>0(R>0)$, then we regard $\lambda_{S_{i}}=+\infty\left(\lambda_{R_{i}}=+\infty\right)$.

Due to these assumptions, the functions $f_{i}(S, R)$ satisfy a Lipschitz condition in $S$ and $R$ on any compact subset of $\mathbb{R}_{+} \times \mathbb{R}_{+}$, and therefore we have uniqueness of initial-value problems and continuous dependence on initial conditions and parameters for the system (2.1).

If there are only two species, then (2.1) is precisely model III in [18] adapted to the chemostat, as well as the model studied in [6] in the non-inhibitory kinetics case. If we further assume that the $p_{i}$ and $q_{i}$, satisfy Michaelis-Menten dynamics, it is the model studied by Hsu, Cheng and Hubbell [15]. By means of linear analysis, Leon and Tumpson [18] provided local analysis of steady states, and pointed out that the necessary and sufficient conditions for coexistence of two species at a locally asymptotically stable steady equilibrium "are that each species must at equilibrium consume greater fraction of the net rate of supply of its limiting resource than of the net rate of supply of its competitor's limiting resource". Hsu, Cheng and Hubbell [15], and Butler and Wolkowicz [6], on the other hand, gave a complete global analysis of the model. 
Following [15], we define

$$
C_{i}=y_{S_{i}} / y_{R_{i}}, \quad i=1,2, \ldots, n
$$

The parameter $C_{i}$ represents the fixed yield ratio for species $i$ growing on $R$ and $S$, since the units of $1 / y_{S_{i}}$ are (units of $S$ consumed)/(units of population $i$ produced).

Let

$$
T_{i}=\frac{R^{0}-\lambda_{R_{i}}}{S^{0}-\lambda_{S_{i}}}, \quad i=1,2, \ldots, n,
$$

and

$$
T_{i j}=\frac{R^{0}-\lambda_{R_{i}}}{S^{0}-\lambda_{S_{j}}}, \quad i, j=1,2, \ldots, n .
$$

The parameter $T_{i}$ represents the ratio in which nutrients $S$ and $R$ are externally regenerated under steady-state consumption pressure from the population $i$ in the absence of its competitors. If $T_{i}>C_{i}$, we say that population $i$ is $S$-limited because $S$ is regenerating at a steady-state rate slower than $R$ with respect to the required consumption ratio of population $i$. Similarly, if $T_{i}<C_{i}$ then we say that population $i$ is $R$-limited. These designations will be clarified below when we determine steady states. $T_{i j}$ represents the ratio of the steady state regeneration rate of $R$ when $x_{i}$ is alone to that of $S$ when $x_{j}$ is alone. Note that $T_{i i}=T_{i}$.

To avoid steady states for which the associated Jacobian matrix of the linearization has any real root equal to zero, if the parameters are finite, we assume that

$$
\begin{aligned}
& \lambda_{S_{i}} \text { and } \lambda_{S_{j}} \text { are distinct from each other and from } S^{0} \\
& \lambda_{R_{i}} \text { and } \lambda_{R_{j}} \text { are distinct from each other and from } R^{0}
\end{aligned}
$$

and

$$
T_{i j} \text { is distinct from } C_{i} \text { and } C_{j} \text {. }
$$

To ensure that the critical points are all isolated we assume that for each $i$ and $j$ with $i \neq j$

$$
C_{i} \neq C_{j}
$$


We further assume that

$$
T_{i} \neq C_{j}
$$

which ensure that if $\left(\bar{S}, \bar{R}, \bar{x}_{1}, \ldots, \bar{x}_{n}\right)$ is a critical point, then $p_{i}(\bar{S}) \neq q_{i}(\bar{R})$ for $i=1,2, \ldots, n$.

We assume the equations are numbered such that

$$
\lambda_{S_{1}}<\lambda_{S_{2}}<\lambda_{S_{3}}<\ldots<\lambda_{S_{n}}
$$

This assumption will help us save space in describing various outcomes of the chemostat without loss of generality. Here we do not make any assumption on the order of $\lambda_{R_{1}}, \lambda_{R_{2}}, \ldots, \lambda_{R_{n}}$.

We now state some preliminary results for system (2.1). It is easy to see that solutions of (2.1) are positive and bounded. Forming linear combinations of the variables, we obtain the following two equations.

$$
\frac{d}{d t}\left\{S(t)+\sum_{i=1}^{n} \frac{x_{i}(t)}{y_{S_{i}}}\right\}=D\left\{S^{0}-S(t)-\sum_{i=1}^{n} \frac{x_{i}(t)}{y_{S_{i}}}\right\},
$$

and

$$
\frac{d}{d t}\left\{R(t)+\sum_{i=1}^{n} \frac{x_{i}(t)}{y_{R_{i}}}\right\}=D\left\{R^{0}-R(t)-\sum_{i=1}^{n} \frac{x_{i}(t)}{y_{R_{i}}}\right\} .
$$

Solving these leads to

$$
S(t)+\sum_{i=1}^{n} \frac{x_{i}(t)}{y_{S_{i}}}=S^{0}+O(\exp (-D t))
$$

and

$$
R(t)+\sum_{i=1}^{n} \frac{x_{i}(t)}{y_{R_{i}}}=R^{0}+O(\exp (-D t)) .
$$

The polygonal set

$$
M=\left\{\left(S, R, x_{1}, \ldots, x_{n}\right) \in R^{n}: S+\sum_{i=1}^{n} \frac{x_{i}}{y_{S_{i}}}=S^{0}, \text { and } R+\sum_{i=1}^{n} \frac{x_{i}}{y_{R_{i}}}=R^{0}\right\}
$$


is an invariant and global attracting set for (2.1). The restriction of (2.1) to $M$ is given by

$$
\begin{aligned}
& x_{i}^{\prime}(t)=x_{i}(t)\left(f_{i}\left(S^{0}-\sum_{i=1}^{n} \frac{x_{i}(t)}{y_{S_{i}}}, R^{0}-\sum_{i=1}^{n} \frac{x_{i}(t)}{y_{R_{i}}}\right)-D\right), \\
& x_{i}(0)>0, \quad i=1,2, \ldots, n
\end{aligned}
$$

on the set

$$
\Gamma=\left\{x \in \mathbb{R}_{+}^{n}: \sum_{i=1}^{n} \frac{x_{i}}{y_{S_{i}}} \leq S^{0}, \sum_{i=1}^{n} \frac{x_{i}}{y_{R_{i}}} \leq R^{0}\right\} .
$$

It is easily seen that the vector field points inward on the boundary of $\Gamma$ implying that $\Gamma$ is positively invariant. The resources may be easily recovered from the equations

$$
S(t)=S^{0}-\sum_{i=1}^{n} \frac{x_{i}(t)}{y_{S_{i}}}, \quad R(t)=R^{0}-\sum_{i=1}^{n} \frac{x_{i}(t)}{y_{R_{i}}} .
$$

System (2.15) is referred as the limiting or reduced system for (2.1).

\section{$3 \quad$ Steady States and Their Local Stability}

The potential steady states are summarized in Table 1. Briefly, the washout steady state contains no organisms and is uninteresting. Potential single-species steady states for population $k$ come in two varieties, at most one of which may exist: $E_{S_{k}}$ when the $k$ th species is $S$-limited, $E_{R_{k}}$ when it is $R$-limited. Occasionally we will drop the $S$ or $R$ and write $E_{k}$ for the single species steady state. There are $n(n-1) / 2$ potential steady states involving coexistence of two species, say species $k$ and $l$ with $k<l$, denoted by $E_{k l}$. No other steady states exist. We summarize notation for the possible steady states in Table 1.

We now discuss criteria for the existence of the steady states. The washout steady state $E_{0}$ always exists. For $E_{S_{k}}$ to exist, we must have $q\left(R^{0}-C_{k}\left(S^{0}-\lambda_{S_{k}}\right)\right)>D$ which is equivalent to $R^{0}-C_{k}\left(S^{0}-\lambda_{S_{k}}\right)>\lambda_{R_{k}}$ or $T_{k}>C_{k}$. Similarly, $E_{R_{k}}$ exists if $T_{k}<C_{k}$. In other words, $E_{S_{k}}$ exists if the species $k$ is $S$-limited, and $E_{R_{k}}$ exists if the species $k$ is $R$-limited. $E_{k l}$ exists if

$$
\begin{aligned}
& R^{0}-\frac{\bar{x}_{k}}{y_{R_{k}}}-\frac{\bar{x}_{l}}{y_{R_{l}}}=\lambda_{R_{k}}, \\
& S^{0}-\frac{\bar{x}_{l}}{y_{S_{l}}}-\frac{\bar{x}_{l}}{y_{S_{l}}}=\lambda_{S_{l}},
\end{aligned}
$$




\begin{tabular}{|c|c|}
\hline Symbol & Steady state \\
\hline$E_{0}$ & $=\left(S^{0}, R^{0}, 0, \ldots, 0\right)$ \\
\hline$E_{S_{k}}$ & $\begin{array}{l}=\left(\lambda_{S_{k}}, r_{k}, 0, \ldots, 0, \bar{x}_{k}, 0, \ldots, 0\right) \\
\text { where } r_{k}=R^{0}-C_{k}\left(S^{0}-\lambda_{S_{k}}\right) \quad \text { and } \quad \bar{x}_{k}=\left(S^{0}-\lambda_{S_{k}}\right) y_{S_{k}}\end{array}$ \\
\hline$E_{R_{k}}$ & $\begin{array}{l}=\left(s_{k}, \lambda_{R_{k}}, 0, \ldots, 0, \bar{x}_{k}, 0, \ldots, 0\right) \\
\text { where } s_{k}=S^{0}-\left(R^{0}-\lambda_{R_{k}}\right) / C_{k} \quad \text { and } \quad \bar{x}_{k}=\left(R^{0}-\lambda_{R_{k}}\right) y_{R_{k}}\end{array}$ \\
\hline$E_{k l}$ & $\begin{array}{l}=\left(\lambda_{S_{l}}, \lambda_{R_{k}}, 0, \ldots, 0, \bar{x}_{k}, 0, \ldots, 0, \bar{x}_{l}, 0, \ldots, 0\right) \\
\text { where } \bar{x}_{k}=y_{S_{k}} y_{R_{k}} \frac{y_{S_{l}}\left(S^{0}-\lambda_{S_{l}}\right)-y_{R_{l}}\left(R^{0}-\lambda_{R_{k}}\right)}{y_{S_{l}} y_{R_{k}}-y_{S_{k}} y_{R_{l}}} \\
\qquad \bar{x}_{l}=y_{S_{l}} y_{R_{l}} \frac{y_{R_{k}}\left(R^{0}-\lambda_{R_{k}}\right)-y_{S_{k}}\left(S^{0}-\lambda_{S_{l}}\right)}{y_{S_{l}} y_{R_{k}}-y_{S_{k}} y_{R_{l}}}\end{array}$ \\
\hline
\end{tabular}

Table 1: Notation for the Steady States

$$
\begin{aligned}
& p_{k}\left(\lambda_{S_{l}}\right)>D, \\
& q_{l}\left(\lambda_{R_{k}}\right)>D .
\end{aligned}
$$

In order for $E_{k l}$ to exist, we must have $\bar{x}_{k}>0$ and $\bar{x}_{l}>0$ which lead to $C_{k}<T_{k l}<C_{l}$ or $C_{k}>T_{k l}>C_{l}$. By (2.11), (3.2) is automatically true. (3.3) holds if and only if $\lambda_{R_{l}}<\lambda_{R_{k}}$

In Table 2, we summarize criteria that ensure that these steady states lie in the nonnegative cone, as well as criteria that guarantee their local asymptotic stability. The linear analysis for local stability of steady states is standard and can be found in Appendix 1. We shall denote the steady states for the reduced system (2.15) by $F_{0}, F_{S_{k}}, F_{R_{k}}$ and $F_{k l}$ that correspond to $E_{0}, E_{S_{k}}, E_{R_{k}}$ and $E_{k l}$ respectively where the nutrient variables are dropped. The criteria in Table 2 also work for the steady states for the reduced system (2.15).

Tilman [36] (see also Grover [8]) developed a graphical analysis which gives insight and will prove to be useful here so we describe it briefly. Consider the positive quadrant of the $S, R$ plane. The isocline for the $i$-th population:

$$
f_{i}(S, R)-D=0
$$

separates the quadrant into two components. It is an $L$-shaped curve with vertex 


\begin{tabular}{|c|c|c|}
\hline Steady state & Criteria for existence & $\begin{array}{l}\text { Criteria for } \\
\text { asymptotic stability }\end{array}$ \\
\hline$\overline{E_{0}}$ & always exists & $\begin{array}{l}\lambda_{S_{i}}>S^{0} \text { or } \lambda_{R_{i}}>R^{0} \\
\text { for } i=1, \ldots, n\end{array}$ \\
\hline$E_{S_{k}}$ & $\lambda_{S_{k}}<S^{0}, \quad \lambda_{R_{k}}<R^{0}, \quad T_{k}>C_{k}$ & $T_{i k}<C_{k} \quad$ for $\quad 1 \leq i<k$ \\
\hline$E_{R_{k}}$ & $\lambda_{S_{k}}<R^{0}, \quad \lambda_{R_{k}}<R^{0}, \quad T_{k}<C_{k}$ & $\begin{array}{l}\lambda_{R_{k}}<\lambda_{R_{i}} \quad \text { or } \quad T_{k i}>C_{k} \\
\text { for } 1 \leq i \leq n \quad \text { and } i \neq k\end{array}$ \\
\hline$E_{k l}$ & $\begin{array}{l}k<l, \lambda_{R_{l}}<\lambda_{R_{k}}<R^{0} \\
\text { and } \\
\left(\begin{array}{c}C_{k}<T_{k l}<C_{l} \\
\text { or } \\
C_{k}>T_{k l}>C_{l}\end{array}\right)\end{array}$ & $\begin{array}{l}C_{k}>C_{l}, \text { and } \lambda_{R_{k}}<\lambda_{R_{i}} \\
\text { for } 1 \leq i<l \text { and } i \neq k\end{array}$ \\
\hline
\end{tabular}

Table 2: Summary of Local Stability

$\left(\lambda_{S_{i}}, \lambda_{R_{i}}\right)$. The point $\left(S^{0}, R^{0}\right)$ is called the supply point (or vector) and

$$
Q_{i}=\left(y_{S_{i}}^{-1}, y_{R_{i}}^{-1}\right)
$$

the consumption vector for population $i$. The existence of a single-population steady state $E_{i}=E_{S_{i}}$ or $E_{R_{i}}$, which for brevity we write as $\left(S_{i}, R_{i}, x_{i}\right)$ ignoring its zero components, requires that

$$
\left(S_{i}, R_{i}\right)=\left(S^{0}, R^{0}\right)-x_{i} Q_{i}, \quad f_{i}\left(S_{i}, R_{i}\right)-D=0
$$

Thus, the supply point must belong to the upper right component, $f_{i}-D>0$ of the quadrant. Note $x_{i}>0$ is uniquely determined by the supply point, the consumption vector, and the requirement that $\left(S_{i}, R_{i}\right)$ belong to the isocline. The figure below depicts the case of population $i$ being $R$-limited $\left(R_{i}=\lambda_{R_{i}}\right)$.

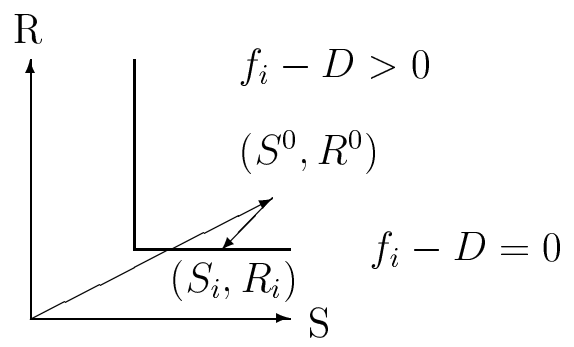


Now consider what our hypotheses (2.7)-(2.11) mean graphically. (2.7) says that the supply point does not belong to any isocline and that every pair of isoclines have at most one point of intersection. (2.9) says that the slopes of the consumption vectors $Q_{i}, i=1, \ldots, n$ are distinct. In particular, the $Q_{i}$ are pairwise linearly independent. Of course, (2.10) is a technical assumption which is crucial because it means that we can linearize about each steady state.

Suppose the $i$ and $j$ isoclines intersect and $i<j$ so $\lambda_{S_{i}}<\lambda_{S_{j}}$. The point of intersection is $\left(\lambda_{S_{j}}, \lambda_{R_{i}}\right)$. Planar steady state $E_{i j}$ can exist only if the supply vector lies in the intersection of each of the outer regions where $f_{i}-D>0$ and $f_{j}-D>0$. If $E_{i j}$ exists, then the nutrient values are given by $(S, R)=\left(\lambda_{S_{j}}, \lambda_{R_{i}}\right)$. It is not hard to see that (2.8) says that neither $\left(S_{i}, R_{i}\right)$ nor $\left(S_{j}, R_{j}\right)$ may coincide with $\left(\lambda_{S_{j}}, \lambda_{R_{i}}\right)$, i.e., the nutrient coordinates of $E_{i}, E_{j}$ and $E_{i j}$ are distinct. Thus we may identify the steady states with their "nutrient coordinates" in the quadrant, ignoring the $x_{i}, x_{j}$ coordinates. Explicitly, we may write $E_{i}=\left(S_{i}, R_{i}\right)$ and $E_{i j}=\left(\lambda_{S_{j}}, \lambda_{R_{i}}\right)$. Now, $E_{i j}$ exists if and only if the supply vector belongs to the cone

$$
C=C_{i j}=\left\{(S, R):(S, R)=\left(\lambda_{S_{j}}, \lambda_{R_{i}}\right)+x_{i} Q_{i}+x_{j} Q_{j}, x_{i}, x_{j}>0\right\}
$$

with vertex at $\left(\lambda_{S_{j}}, \lambda_{R_{i}}\right)$. Indeed, if the supply point belongs to $C$, then (here we include $x_{i}, x_{j}$ in the notation)

$$
E_{i j}=\left(\lambda_{S_{j}}, \lambda_{R_{i}}, x_{i}, x_{j}\right)
$$

and

$$
\left(S^{0}, R^{0}\right)=\left(\lambda_{S_{j}}, \lambda_{R_{i}}\right)+x_{i} Q_{i}+x_{j} Q_{j} .
$$

This has a nice graphical interpretation as shown below. 


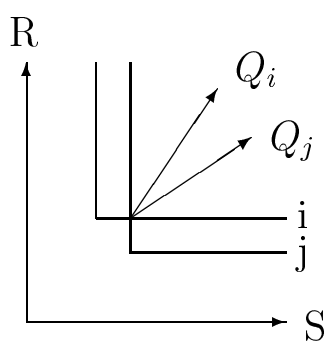

It is not hard to see that the stability of $E_{i j}$ as a steady state of the twopopulation model where only species $i$ and $j$ are present in (2.1) is determined by the relative slopes of the consumption vectors $Q_{i}$ and $Q_{j}$. $E_{i j}$ is asymptotically stable if $Q_{i}$ has greater slope than does $Q_{j}$ (as depicted in the figure above) and it is a saddle point if the reverse relation of slopes holds. Keep in mind that we assume that the supply point belongs to the cone $C$ and that $\lambda_{S_{i}}<\lambda_{S_{j}}$.

Finally, if $E_{i j}$ exists, it is easy to determine whether it is stable or unstable to invasion by species $k \neq i, j$ from the relative configuration of the isoclines in the quadrant. We want to know if $x_{k}$ can invade $E_{i j}$, that is, whether $f_{k}-D>0$ at $E_{i j}$ or whether the reverse inequality holds so $x_{k}$ cannot invade. If $E_{i j}$ belongs to the upper right component of the $k$ th isocline then $x_{k}$ can invade while if it belongs to the lower left component, then it cannot. Of course, the same analysis applies to $E_{i}$. Consequently, much information is provided by the relative configurations of the isoclines.

\section{Main Results}

The dynamics of the system (2.1) is studied in this section. We are interested in establishing global asymptotic stability for one of the steady states and in determining when a species is doomed to extinction. The general case is considered in section 4.1 while the special case $n=3$ is treated in 4.2 . Proofs are deferred to section 6 .

\subsection{Main results for general $n \geq 2$}

Before stating our main results, we begin by identifying some cases where a given 
species or set of species are doomed to extinction. The first theorem in this section says that if the input concentration of either (or both) nutrient is inadequate for a given population, then it will be washed out of the chemostat.

Theorem 4.1 If $\lambda_{S_{i}}>S^{0}$ or $\lambda_{R_{i}}>R^{0}$ for some $i$, then $x_{i}(t) \rightarrow 0$ as $t \rightarrow \infty$ for (2.1).

Hereafter, we only consider populations for which there is adequate nutrient. Explicitly, we assume that $\lambda_{S_{i}}<S^{0}$ and $\lambda_{R_{i}}<R^{0}$ for all $i$.

There are other scenarios where extinctions are not due to insufficient resources but to competitive effects. If $\lambda_{R_{1}}<\lambda_{R_{i}}$ for $i>1$ then species one is the superior competitor on both resources and therefore species $i>1$ are doomed, as indicated below in Corollary 4.1. Another scenario where inferior competitors are apparent is the following.

Theorem 4.2 Suppose that for some $k>1$,

$$
\lambda_{R_{n}}>\lambda_{R_{n-1}}>\ldots>\lambda_{R_{k}}>\lambda_{R_{j}}, \quad j<k \text {. }
$$

Then $x_{i}(t) \rightarrow 0$ for $k \leq i \leq n$ for every solution of (2.1) with $x_{j}(0)>0$ for some $j<k$.

More generally, we conjecture that if the $j$ th species isocline is contained in the upper right component of the complement of the $k$ th species isocline, then $x_{j}(t) \rightarrow 0$ for all solutions of $(2.1)$ with $x_{i}(0)>0$ for all $i$. If true, then we could restrict our attention to the case that every isocline meets every other isocline in the $S, R$ plane, i.e.,

$$
\lambda_{R_{n}}<\lambda_{R_{n-1}}<\ldots<\lambda_{R_{1}}
$$


Phillips principle for equilibrium coexistence [25] requires that for each population, there must exist at least one set of resource availabilities at which only this population can grow while others cannot. Although equilibrium coexistence is impossible here for $n>2$, the inequality above expresses his principle. The reader is invited to sketch the resulting isocline configuration in the $S, R$ plane (see Case IV for the case $n=3$ in section 4.2). As support for the conjecture we offer the following simple proposition which says that under the hypotheses of the conjecture, either $x_{j}(t) \rightarrow 0$ or it oscillates and in the later case, the same alternative applies to $x_{k}$.

Proposition 4.1 If $f_{k}-D>0$ at all points $(S, R) \in \mathbb{R}_{+}^{2}$ where $f_{j}-D=0$ then either $\lim x_{j}(t)=0$ or $\liminf x_{j}(t)<\limsup x_{j}(t)$. In the latter case, either $x_{k}(t) \rightarrow 0$ or $\liminf x_{k}(t)<\limsup x_{k}(t)$.

Our main results for general $n \geq 2$ follow. The first two provide conditions for species $k$ to be the only survivor in the chemostat. There are two possibilities: it is $S$-limited or it is $R$-limited. The first result considers the $S$-limited case.

Theorem 4.3 Assume that $T_{k}>C_{k}$ for some $k, 1 \leq k \leq n$ and, in addition, that (i) for each $j$ with $1 \leq j<k, T_{j k}<C_{i}$ for $1 \leq i \leq n$;

(ii) for each $j$ with $k<j \leq n$, either $\lambda_{R_{k}}<\lambda_{R_{j}}$ or $T_{k j}>C_{i}$ for $1 \leq i \leq n$. Then $E_{S_{k}}$ attracts all solutions with $x_{k}(0)>0$ for (2.1).

The hypotheses of Theorem 4.3 have a number of less obvious implications. If $j<k$, and if $\lambda_{R_{j}}<\lambda_{R_{k}}$ then $T_{j k}>T_{k}>C_{k}$, contradicting (i). Thus, $\lambda_{R_{j}}>\lambda_{R_{k}}$ for $j<k$. Furthermore, it can easily be seen that for $1 \leq j<k, x_{j}$ is $R$-limited (i.e., $T_{j}<C_{j}$ ), and for $k \leq j \leq n, x_{j}$ is $S$-limited (i.e., $T_{j}>C_{j}$ ). Now, as species $k$ has the lowest break-even concentration for resource $R$ among the $R$-limited strains 1 , $\ldots, k-1$, and it has the lowest break-even concentration for resource $S$ among the $S$-limited strains $k, \ldots, n$, population $k$ always wins. 
The parallel result for $R$-limited species $k$ is stated below.

Theorem 4.4 Let $T_{k}<C_{k}$ for some $k$. Assume that

(i) for $k<j \leq n$, either $\lambda_{R_{k}}<\lambda_{R_{j}}$ or $T_{k j}>C_{i}$ for $1 \leq i \leq n$;

(ii) for $1 \leq j<k, T_{j k}<C_{i}$ for $1 \leq i \leq n$.

Then $E_{R_{k}}$ attracts all solutions with $x_{k}(0)>0$ for (2.1).

We would like to point out that the conditions in Theorem 4.3 and Theorem 4.4 are necessary if there are only two species in the chemostat. See $[6,15]$ for details. The following corollary of Theorem 4.3 and Theorem 4.4 states that if one species has the lowest break-even concentrations for both nutrients $S$ and $R$, then that species will survive and its rivals will not.

Corollary 4.1 Assume that

$$
\lambda_{R_{1}}<\lambda_{R_{i}} \text { for } 2 \leq i \leq n \text {. }
$$

If $T_{k}>C_{k}\left(T_{k}<C_{k}\right)$, then $E_{S_{1}}\left(E_{R_{1}}\right)$ attracts all solutions with $x_{k}(0)>0$ for (2.1).

As we see from Table 2, $E_{k l}$ is locally asymptotic stable if and only if $\lambda_{R_{i}}>$ $\lambda_{R_{k}}>\lambda_{R_{l}}$ for $i<l, i \neq k$ as well as $C_{k}>T_{k l}>C_{l}$. In this case, we arrange $\lambda_{R_{1}}$, $\lambda_{R_{2}}, \ldots, \lambda_{R_{l}}$ in the order

$$
\lambda_{R_{l}}<\lambda_{R_{k}}<\lambda_{R_{k_{1}}}<\lambda_{R_{k_{2}}}<\ldots<\lambda_{R_{k_{l-2}}} .
$$

The next theorem presents conditions for $E_{k l}$ to be a global attractor.

Theorem 4.5 Assume that

(i) $\lambda_{R_{i}}>\lambda_{R_{k}}>\lambda_{R_{l}}$ for $i<l, i \neq k, C_{k}>T_{k l}>C_{l}$, and $\lambda_{R_{1}}$ through $\lambda_{R_{l}}$ are arranged in the order described in (4.2);

(ii) for all $l<j \leq n, T_{h j}>C_{i}$ for $1 \leq h \leq j$ and $1 \leq i \leq j$;

(iii) for all $1 \leq j \leq l-2, T_{k_{j} p}<C_{k}, C_{l}, C_{k_{i}}$, for $p=k, l, k_{m}$ where $1 \leq i, m \leq j$.

Then $E_{k l}$ attracts all solutions with $x_{k}(0) x_{l}(0)>0$ for (2.1). 
Condition (i) in Theorem 4.5 shows that $\lambda_{R_{l}}$ is smallest and $\lambda_{R_{k}}$ is the second smallest among $\lambda_{R_{1}}$ through $\lambda_{R_{l}}$, species $k$ is $R$-limited, and species $l$ is $S$-limited. Condition (ii) implies that for $l<j \leq n$, species $j$ is $S$-limited, and condition (iii) implies that for $1 \leq j<l, j \neq k$, species $j$ is $R$-limited. Therefore species $k$ and $l$ win and the others are washed out. On the other hand, species $k(l)$ has the lower break-even concentration for nutrient $R(S)$ than species $l(k)$. Coexistence of species $k$ and $l$ occurs because each species has the lower break-even concentration for that resource which, at the two-species steady state mixture of nutrients, limits the growth of the other.

\subsection{Main results for $n=3$}

For the special case $n=3$, our results give an essentially complete picture of the dynamics. The mathematical reason for this is that there exists a PoincaréBendixson theory for three dimensional competitive systems [11, 32], in our case, (2.15), and because we can rule out periodic orbits (see $[32,33])$.

Theorem 4.6 If $n=3$, then every solution of (2.1) converges to one of the steady states $E_{i}$ or $E_{i j}$. In particular, at least one species is washed out.

Unfortunately, there are too many possible phase portraits to catalog all of them. We content ourselves by making some general statements and considering a few interesting cases. In order to further describe the dynamics, it is useful to catalog the various possible isocline configurations. There are 6 possible configurations of the isoclines. We label these configurations as follows: (I) $\lambda_{R_{1}}<\lambda_{R_{2}}<\lambda_{R_{3}}$. (II) $\lambda_{R_{1}}<\lambda_{R_{3}}<\lambda_{R_{2}}$. (III) $\lambda_{R_{2}}<\lambda_{R_{1}}<\lambda_{R_{3}}$. (IV) $\lambda_{R_{3}}<\lambda_{R_{2}}<\lambda_{R_{1}}$. $\lambda_{R_{2}}<\lambda_{R_{3}}<\lambda_{R_{1}}$. (VI) $\lambda_{R_{3}}<\lambda_{R_{1}}<\lambda_{R_{2}}$. They are depicted below. 

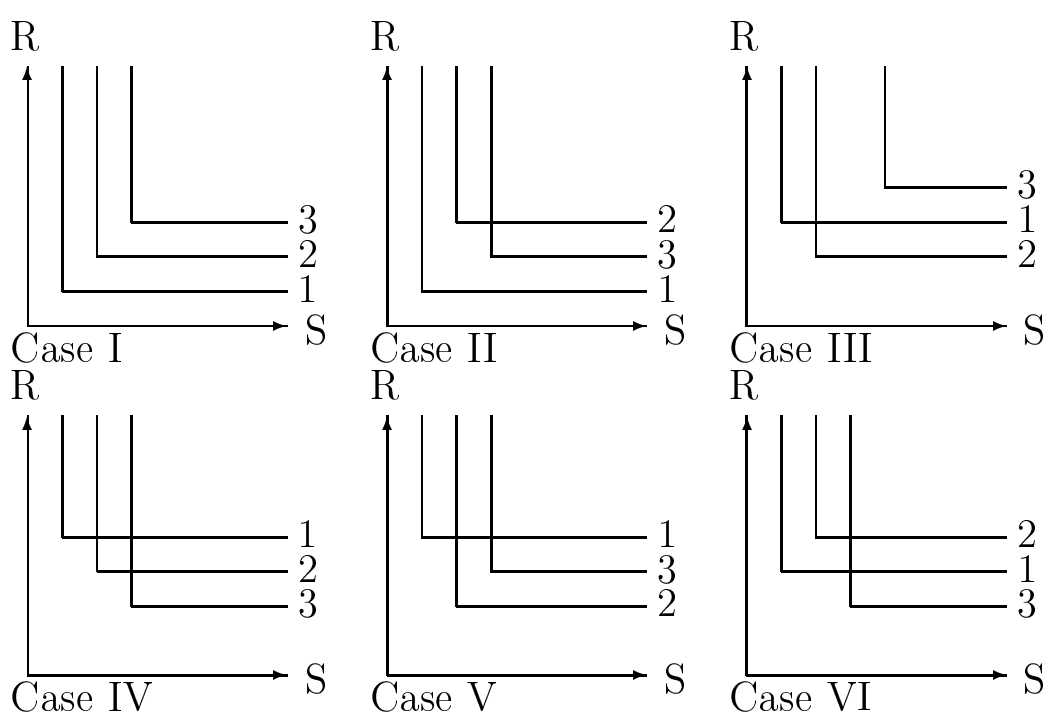

In cases I and II, Corollary 4.1 ensures that species 1 is the winner. Species 3 is washed out in case III by Theorem 4.2; it is also washed out in case V by Proposition 4.1 and Theorem 4.6. Species 2 is washed out in case VI for similar reasons. Except for case I, there are several phase portraits that are possible; for cases II,III,V,VI, these are determined by results for the case $n=k=2$ (see Hubbell et al [15]). Clearly, case IV is the most interesting since there are no obvious extinctions.

It is crucial for our proof of Theorem 4.6 that there are no heteroclinic cycles of steady states. Recall that a heteroclinic cycle of steady states consists of a finite set of equilibria and entire orbits: $E^{1} \rightarrow E^{2} \rightarrow \ldots E^{k} \rightarrow E^{1}$ where the $E^{i}$ are steady states and the arrows indicate heteroclinic orbits. The main ideas of the proof use the Poincaré-Bendixson theory for three dimensional competitive systems and proving the non-existence of a periodic orbit. For case IV, there can be up to three asymptotically stable steady states. In this case, for each population, there exists an asymptotically stable steady state where that population is present. 


\section{Discussion}

In this paper we studied the classical model of $n$ species of microorganisms competing for two perfectly complementary, growth-limiting nutrients in a chemostat. We assumed a general class of monotone increasing response functions for nutrient uptake. Our main results generalize to $n>2$ some results in Hsu, Cheng and Hubbell [15] and in Butler and Wolkowicz [6] who treated the case $n=2$. These results provide conditions which ensure the survival of only one species and the coexistence of two species and they are sharp if there are only two species. We may summarize them as follows. Sufficient conditions for species $k$ to be the sole survivor, given that it is $S$ limited, are roughly that it have the lowest break-even concentration for resource $R$ among the $R$-limited strains $1, \ldots, k-1$, and it has the lowest break-even concentration for resource $S$ among the $S$-limited strains $k, \ldots, n$. Similar conditions suffice when species $k$ is $R$ limited.

In order for species $k$ and $l$ to coexist, the others being washed out, we require that for $l<j \leq n$, species $j$ is $S$-limited, and for $1 \leq j<l, j \neq k$, species $j$ is $R$-limited. Therefore species $k$ and $l$ win and the others are washed out. On the other hand, species $k(l)$ has the lower break-even concentration for nutrient $R(S)$ than species $l(k)$. Coexistence of species $k$ and $l$ occurs because each species has the lower break-even concentration for that resource which, at the two-species steady state mixture of nutrients, limits the growth of the other.

Our results for $n=3$ are essentially complete, every solution of (2.1) converges to one of the steady states $E_{i}$ or $E_{i j}$, implying the extinction of at least one species. In this case, we have an answer to the question in the title: no more than two. It is very difficult to list all possible phase diagrams in terms of explicit parameters, because there are too many of them (approximately forty three cases). There are no heteroclinic cycles of steady states. There can be up to three asymptotically 
stable steady states for a given set of parameters and in this case, every species is represented (has positive density) in at least one of these.

However, for $n>3$, it is possible that a heteroclinic cycle of steady states exists. It can be proved that for the limiting system (2.15) and $n=4$, there exist heteroclinic cycles $F_{1} \rightarrow F_{4} \rightarrow F_{3} \rightarrow F_{2} \rightarrow F_{1}$ and $F_{1} \rightarrow F_{2} \rightarrow F_{4} \rightarrow F_{3} \rightarrow F_{1}$. The criterion for the former cycle to exist is either $\lambda_{R_{4}}<\lambda_{R_{3}}<\lambda_{R_{1}}<\lambda_{R_{2}}, T_{23}>C_{2}, C_{3}, T_{14}<C_{1}, C_{4}$, and $T_{34}>C_{3}, C_{4}$, or $\lambda_{R_{3}}<\lambda_{R_{4}}<\lambda_{R_{1}}<\lambda_{R_{2}}, T_{23}>C_{2}, C_{3}$, and $T_{14}<C_{1}, C_{4}$; the criterion for the later cycle to exist is either $\lambda_{R_{4}}<\lambda_{R_{3}}<\lambda_{R_{2}}<\lambda_{R_{1}}, T_{12}<C_{1}, C_{2}$, $T_{13}>C_{1}, C_{3}, T_{24}<C_{2}, C_{4}$, and $T_{34}>C_{3}, C_{4}$, or $\lambda_{R_{3}}<\lambda_{R_{4}}<\lambda_{R_{2}}<\lambda_{R_{1}}, T_{12}<$ $C_{1}, C_{2}, T_{13}>C_{1}, C_{3}$, and $T_{24}<C_{2}, C_{4}$. Figure 1 shows the existence of a cycle of steady states for a particular case of system (2.15) when $n=4$ and $p_{i}$ and $q_{i}$ take Michaelis-Menten form $\left(p_{i}(S)=m_{i} S /\left(a_{i}+S\right)\right.$ and $\left.q_{i}(R)=n_{i} S /\left(b_{i}+S\right)\right)$.

Simulations for the system described in Figure 1 show that solutions approach $E_{23}$ (Figure 7 and Figure 8). The mathematical significance of a heteroclinic cycle is that it represents a potential limit set, if not for an orbit of the particular system, then possibly for an orbit of a larger system obtained by adding more species to this one. Furthermore, a cycle of steady states may spawn the bifurcation of a long-period periodic orbit. Another consequence of the existence of heteroclinic cycles is that understanding the behavior of the orbits of (2.1) for a given $n$ may not be sufficient to understand the behavior of orbits of a larger system obtained by adding more species. Let us elaborate on this a bit. One strategy for obtaining a convergence result for our system is to proceed by induction since the cases $n=2,3$ are settled. In this approach, one would seek to prove that some species becomes extinct and therefore solutions would approach orbits of the lower dimensional system. The problem is that solutions could approach a heteroclinic cycle of the lower dimensional system (even though this cycle is not the limit set of any orbit of the lower dimensional 


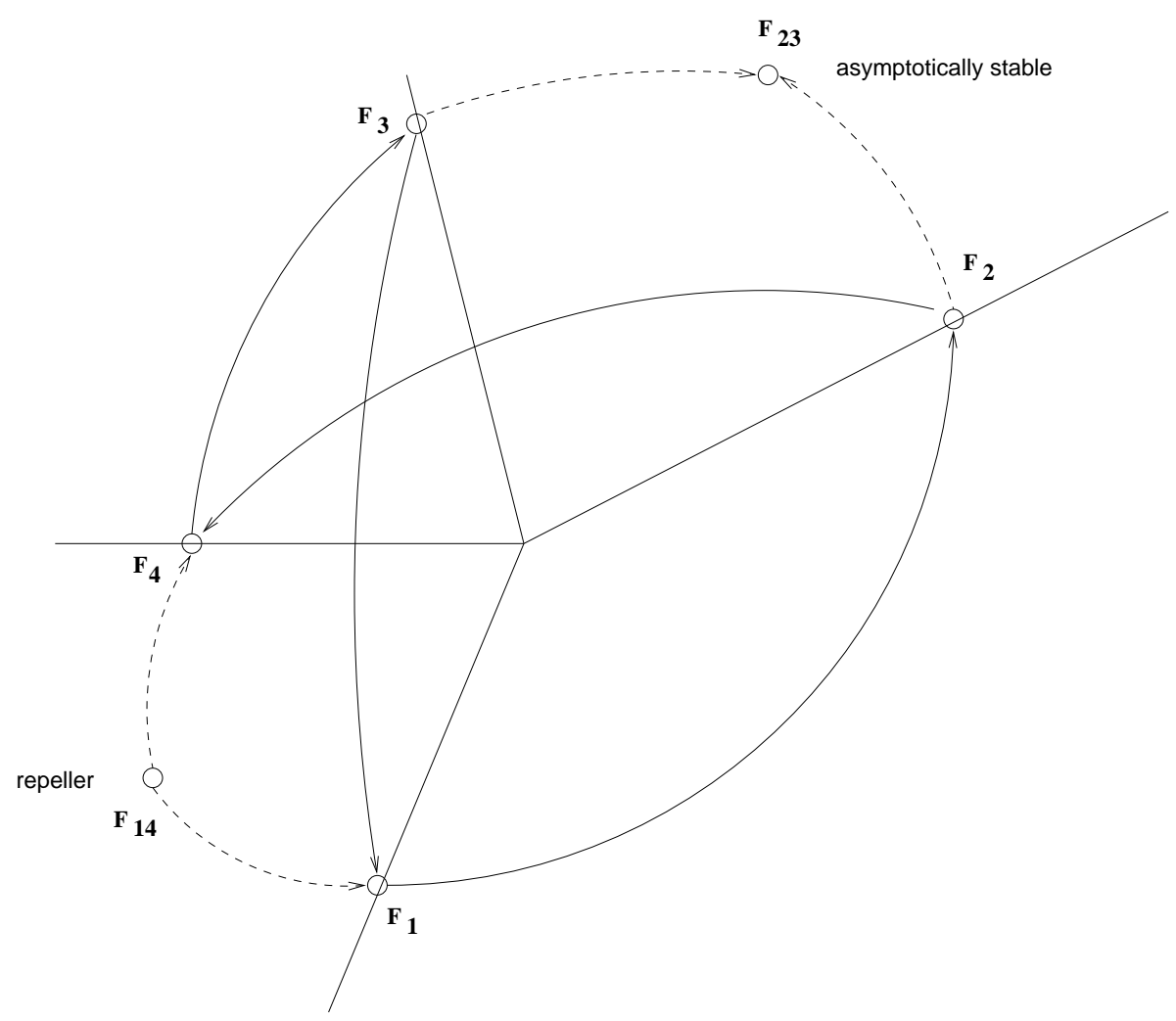

Figure 1: $\quad D=1, S^{0}=1, R^{0}=1.5, a_{i}=b_{i}=1$ for $1 \leq i \leq 4, m_{1}=9, m_{2}=3.5$, $m_{3}=2.66, m_{4}=2.25, n_{1}=1.8, n_{2}=1.9, n_{3}=6, n_{4}=4, y_{S_{i}}=1$ for $1 \leq i \leq 4$, $y_{R_{1}}=2, y_{R_{2}}=0.3, y_{R_{3}}=2.3, y_{R_{4}}=0.2$. It is easily verified that there is a cycle $F_{1} \rightarrow F_{2} \rightarrow F_{3} \rightarrow F_{4} \rightarrow F_{1}$ lying in the sub-planes $x_{i} x_{j}$ on the boundary of $\mathbb{R}^{4}$ where $F_{i}$ is either $F_{S_{i}}$ or $F_{R_{i}}$.

system). The May-Leonard system [23] provides an example that heteroclinic cycles may arise in Lotka-Volterra competitive systems when $n=3$. From a biological perspective, an orbit approaching a heteroclinic cycle of equilibria is less significant because one expects extinction when one of the population densities becomes, and remains for a very long time, near zero.

We have not answered the question in the title in the general case. Our simulations have invariably suggested that convergence to steady state is the rule; there is no sign of the oscillations seen in [17] for the case $n \geq k=3$. They saw a periodic solution when $n=3$ where species one peaks, followed by species two, then species 
three and so on. It can be proved that no such solution can exist in the case of two resources. Yet we cannot rule out oscillations with more complicated patterns of extrema. We present a few simulations below. Consider system (2.1) when $n=5$, $D=1, S^{0}=1, R^{0}=1.5$, and $p_{i}(S)=m_{i} S /\left(a_{i}+S\right)$ and $q_{i}(R)=n_{i} S /\left(b_{i}+S\right)$ where $a_{i}=b_{i}=1$ for $1 \leq i \leq 5, m_{1}=9, m_{2}=3.5, m_{3}=2.66, m_{4}=2.25, m_{5}=2.1$, $n_{1}=1.8, n_{2}=1.9, n_{3}=4, n_{4}=6, n_{5}=11, y_{S_{i}}=1$ for $1 \leq i \leq 5, y_{R_{1}}=2$, $y_{R_{2}}=0.3, y_{R_{3}}=2.3, y_{R_{4}}=0.06$, and $y_{R_{5}}=0.6$. One can verify that each $E_{i}$ is unstable, there are four coexistence steady states: $E_{15}, E_{23}, E_{34}$, and $E_{45}$ with the properties that $E_{15}$ is a repeller, $E_{23}$ and $E_{45}$ are asymptotically stable, and $E_{34}$ is a saddle with the dimension of the stable manifold being 2 ( $E_{34}$ is also a saddle in the $x_{3}-x_{4}$ sub-plane). Extensive simulations show solutions approach either $E_{23}$ (Figure 9 and Figure 10) or $E_{45}$ (Figure 11 and Figure 12).

Butler and Wolkowicz [6] show that oscillations occur for the case in which at least one response function for nutrient uptake has two nontrivial break-even concentrations due to growth-inhibition at high nutrient concentration. It would be interesting to investigate this problem when more species are involved in the chemostat. We leave this problem for future investigation.

\section{Proofs}

\subsection{General $n>2$}

The proof of Theorem 4.1, an elementary application of differential inequalities, is essentially the same as in Hubbell et al [15] so we do not give it here.

The following lemma is very useful. A proof can be found in [31].

Lemma 6.1 (Butler-McGehee lemma). Suppose that $p$ is a hyperbolic steady state of (2.1) or (2.15) with the property that $p \in \omega(x)$ and $p \neq \omega(x)$ where $\omega(x)$ is the omega limit set of a solution initiating at $x$ that has nonnegative components. 
Then $\omega(x)$ has nontrivial (i.e., different from $p$ ) intersection with the stable and the unstable manifolds of $p$.

Throughout this section, $W^{s}(p)$ and $W^{u}(p)$ denotes the stable and unstable manifolds of $p$ respectively.

Proof of Theorem 4.2. Let $\Omega$ be the omega limit set of such a solution. We first show that $y_{n}=0$ for all $(\bar{S}, \bar{R}, y) \in \Omega$. If $\lim x_{n}(t)$ exists and is positive, then $f_{n}(S(t), R(t))-D \rightarrow 0$. But this implies that $f_{j}-D>0$ for $j<n$ for all large $t$ so $x_{j}^{\prime}(t) \geq 0$. Consequently, $\lim x(t)$ exists and from this we conclude that $\lim (S(t), R(t), x(t))$ exists and is a steady state with $x_{i}>0$ and $x_{n}>0$. As no such steady state exists, it follows that $\lim x_{n}(t)$ vanishes if it exists. If it doesn't exist, then there is a point $q=(\bar{S}, \bar{R}, y) \in \Omega$ with $y_{n}=\limsup x_{n}(t)>0$ and $f_{n}(\bar{S}, \bar{R})-D=0$. Further, the omega limit point $q$ is not the equilibrium $E_{S_{n}}$ or $E_{R_{n}}$ because the Butler McGehee lemma would imply that $\Omega$ would also contain a point $p \in M$ on the stable manifold of the equilibrium and distinct from it. By our hypotheses, the intersection of $M$ and the stable manifold consists of points satisfying $x_{j}=0,1 \leq j \leq n-1$ and the backward orbit through $p$, also belonging to $\Omega \cap M$, either leaves $\mathbb{R}^{n+2}$ or connects the single species steady state with $E_{0}$, either way we have a contradiction. Thus, $y_{i}>0$ for some $i$ other than $n$. The solution through $q$ is given by $(\bar{S}(t), \bar{R}(t), y(t))$ where $y(0)=y, y(t)$ satisfies the limiting equation $(2.15), \bar{S}(t)=S^{0}-\sum_{i=1}^{n} \frac{y_{i}(t)}{y_{S_{i}}}$ and $\bar{R}(t)=R^{0}-\sum_{i=1}^{n} \frac{y_{i}(t)}{y_{R_{i}}}$. As $f_{n}(\bar{S}(0), \bar{R}(0))-D=0$ and $y_{i}(0)>0$, we see that $\bar{S}^{\prime}(0)=-\sum_{j=1}^{n} \frac{y_{j}^{\prime}(0)}{y_{S_{j}}}<0$ and $\bar{R}^{\prime}(0)<0$. Therefore, $f_{n}(\bar{R}(h), \bar{S}(h))-D>0$ for small $h<0$ so $y_{n}(h)>$ $y_{n}(0)$. But this contradicts that $(\bar{S}(h), \bar{R}(h), y(h)) \in \Omega$ which implies that $y_{n}(h) \leq$ $\limsup x_{n}(t)=y_{n}(0)$. We conclude that $\lim x_{n}(t)=0$.

Suppose that $k \leq n-1$ as otherwise, we are done. If $\lim x_{n-1}(t)$ exists and is positive then $f_{n-1}-D \rightarrow 0$. It follows that $x_{j}^{\prime}(t) \geq 0$ for all $j<n-1$ and 
large $t$ so $(S(t), R(t), x(t))$ converges to a steady state (since $S$ and $R$ must also converge). As $x_{j}(0)>0$ for some $j<k$ we conclude that the steady state has $x_{j}, x_{n-1}>0$. But no such steady state exists. Thus, if $\lim x_{n-1}(t)$ exists, it vanishes. Suppose the limit doesn't exist. Then there is a point $q=(\bar{S}, \bar{R}, y) \in \Omega$ with $y_{n-1}=\limsup x_{n-1}>0$ and $f_{n-1}(\bar{S}, \bar{R})-D=0$. Now $q$ cannot be $E_{R_{n-1}}$ or $E_{S_{n-1}}$ because by the Butler McGehee lemma, $\Omega$ would have to contain a point of its unstable manifold other than the steady state itself. But by our hypotheses, the unstable manifold (one dimensional) has the property that all its accessible points (those points with non-negative coordinates) other than the steady state itself have positive $n$-th coordinates. Since no such point can belong to $\Omega, q$ cannot be $E_{R_{n-1}}$ or $E_{S_{n-1}}$. Therefore, $y_{i}(0)>0$ for some $i<n-1$. Consider the solution $\left(\bar{S}(t), \bar{R}(t), y(t)\right.$ starting at $q$, the orbit of which must belong to $\Omega$. $\bar{S}(t)=S^{0}-$ $\sum_{i=1}^{n} \frac{y_{i}(t)}{y_{S_{i}}}$ satisfies $\bar{S}^{\prime}(0)=-\sum_{i=1}^{n} \frac{y_{i}^{\prime}(0)}{y_{S_{i}}}<0$ because some $y_{i}(0)>0, y_{n}(0)=0$ and $f_{n-1}(\bar{S}(0), \bar{R}(0))-D=0$. Similarly, $\bar{R}^{\prime}(0)<0$. Consequently, $f_{n-1}(\bar{S}(h), \bar{R}(h))-$ $D>0$ for small $h<0$ so $y_{n-1}(h)>y_{n-1}(0)$. But $\left(\bar{S}(h), \bar{R}(h), y(h) \in \Omega\right.$ so $y_{n-1}(h) \leq$ $y_{n-1}(0)=\lim \sup y_{n-1}$. This contradiction proves that $\lim x_{n-1}(t)=0$. Clearly, we may continue this reasoning to get the desired conclusion, using the Butler McGehee theorem, because the unstable manifold of $E_{R_{j}}$ or $E_{S_{j}}$ (whichever exists) has the property that all its accessible points other than the steady state itself have at least one positive coordinate with index exceeding $j$ if $j \geq k$.

Proof of Proposition 4.1. If the limit exists and is positive, then $f_{j}-D \rightarrow 0$ and hence there exists $a>0$ such that $f_{k}-D \geq a$ for large $t$. It follows that $x_{k}(t) \rightarrow \infty$ which is impossible. Similarly, if $x_{k}(t)$ has a positive limit as $t \rightarrow \infty$, then $f_{k}-D \rightarrow 0$ so $f_{j}-D<-a$ for some positive constant and all large $t$, which implies $x_{j}(t) \rightarrow 0$.

In order to prove Theorem 4.3 and Theorem 4.4, we shall make use of the reduced 
system (2.15). The following two lemmas show that the conditions of Theorem 4.3 and Theorem 4.4 ensure that $F_{S_{k}}$ and $F_{R_{k}}$ are globally asymptotically stable for $(2.15)$.

Lemma 6.2 If the conditions of Theorem 4.3 are satisfied, then $F_{S_{k}}$ attracts all solutions of (2.15) with $x_{k}(0)>0$.

Lemma 6.3 If the conditions of Theorem 4.4 are satisfied, then $F_{R_{k}}$ attracts all solutions of (2.15) with $x_{k}(0)>0$.

The proof of Lemma 6.2 involves the following twos lemmas that are elementary but useful, the proofs for which can be found in [10, 37].

Lemma 6.4 (Barbălat lemma). Let a be a finite number and $f:[a,+\infty) \rightarrow R$ be $a$ differentiable function. If $\lim _{t \rightarrow \infty} f(t)$ exists (finite) and $f^{\prime}$ is uniformly continuous on $(a,+\infty)$, then $\lim _{t \rightarrow \infty} f^{\prime}(t)=0$.

Lemma 6.5 (Fluctuation lemma). Let a be a finite number and $f:[a,+\infty) \rightarrow R$ be a differentiable function. If $\liminf _{t \rightarrow \infty} f(t)<\lim _{\sup _{t \rightarrow \infty}} f(t)$, then there exist sequences $\left\{t_{m}\right\} \uparrow \infty$ and $\left\{s_{m}\right\} \uparrow \infty$ such that

$$
\begin{aligned}
& \lim _{m \rightarrow \infty} f\left(t_{m}\right)=\limsup _{t \rightarrow \infty} f(t), \quad f^{\prime}\left(t_{m}\right)=0, \\
& \lim _{m \rightarrow \infty} f\left(s_{m}\right)=\liminf _{t \rightarrow \infty} f(t), \quad f^{\prime}\left(s_{m}\right)=0 .
\end{aligned}
$$

Note that solutions of (2.1) or (2.15) are nonnegative and bounded. It follows that the derivatives $S^{\prime}, R^{\prime}, x_{i}^{\prime}, S^{\prime \prime}, R^{\prime \prime}$, and $x_{i}^{\prime \prime}$ (when it exists) for (2.1) or (2.15) are all bounded, and hence $S^{\prime}, R^{\prime}$ and $x_{i}^{\prime}$ are uniformly continuous on $[0,+\infty)$.

The following lemma contains some useful inequalities which will be used repeatedly in the next few proofs. 
Lemma 6.6 Let $j, k$ be fixed.

(a) If $T_{j k}<C_{i}\left(T_{j k}>C_{i}\right)$ for $1 \leq i \leq n$, then there exists $\epsilon>0$ such that whenever $x_{i} \geq 0$ and

$$
S^{0}-\sum_{i=1}^{n} \frac{x_{i}}{y_{S_{i}}} \leq \lambda_{S_{k}} \quad\left(S^{0}-\sum_{i=1}^{n} \frac{x_{i}}{y_{S_{i}}} \geq \lambda_{S_{k}}\right)
$$

there follows

$$
R^{0}-\sum_{i=1}^{n} \frac{x_{i}}{y_{R_{i}}} \leq \lambda_{R_{j}}-\epsilon \quad\left(R^{0}-\sum_{i=1}^{n} \frac{x_{i}}{y_{R_{i}}} \geq \lambda_{R_{j}}+\epsilon\right) .
$$

(b) If $T_{k j}>C_{i}\left(T_{k j}<C_{i}\right)$ for $1 \leq i \leq n$, then there exists $\epsilon>0$ such that whenever $x_{i} \geq 0$ and

$$
R^{0}-\sum_{i=1}^{n} \frac{x_{i}}{y_{R_{i}}} \leq \lambda_{R_{k}} \quad\left(R^{0}-\sum_{i=1}^{n} \frac{x_{i}}{y_{R_{i}}} \geq \lambda_{R_{k}}\right)
$$

there follows

$$
S^{0}-\sum_{i=1}^{n} \frac{x_{i}}{y_{S_{i}}} \leq \lambda_{S_{j}}-\epsilon \quad\left(S^{0}-\sum_{i=1}^{n} \frac{x_{i}}{y_{S_{i}}} \geq \lambda_{S_{j}}+\epsilon\right) .
$$

Proof. We prove the first assertion; the others are proved similarly.

$$
\begin{aligned}
R^{0}-\sum_{i=1}^{n} \frac{x_{i}}{y_{R_{i}}} & =R^{0}-\lambda_{R_{j}}-\sum_{i=1}^{n} \frac{x_{i}}{y_{R_{i}}}+\lambda_{R_{j}} \\
& <\left(S^{0}-\lambda_{S_{k}}\right) \min _{l}\left\{\frac{y_{S_{l}}}{y_{R_{l}}}\right\}-\epsilon-\sum_{i=1}^{n} \frac{x_{i}}{y_{R_{i}}}+\lambda_{R_{j}} \\
& \leq \min _{l}\left\{\frac{y_{S_{l}}}{y_{R_{l}}}\right\} \sum_{i=1}^{n} \frac{x_{i}}{y_{S_{i}}}-\epsilon-\sum_{i=1}^{n} \frac{x_{i}}{y_{R_{i}}}+\lambda_{R_{j}} \\
& \leq \lambda_{R_{j}}-\epsilon .
\end{aligned}
$$

Proof of Lemma 6.2 Let $x(t)=\left(x_{1}(t), \ldots, x_{n}(t)\right)$ be a solution of $(2.15)$ on $M$ determined by (2.14) with $x_{j}(0)>0$ for at least one value of $j$ in addition to $k$, the result being obvious otherwise. Consider $S(t)$ and $R(t)$ given by $(2.16)$. Assume that for some $t_{0} \geq 0, S\left(t_{0}\right) \leq \lambda_{S_{k}}$. It follows immediately $x_{k}^{\prime}\left(t_{0}\right) \leq 0$ and $x_{j}^{\prime}\left(t_{0}\right)<0$ for $j>k$ if $x_{j}(0)>0$ since $\lambda_{S_{k}}<\lambda_{S_{j}}$. By (i) and Lemma 6.6, if 
$S(t)=S^{0}-\sum_{i=1}^{n} \frac{x_{i}(t)}{y S_{i}} \leq \lambda_{S_{k}}$, then there exists a positive constant $\alpha>0$, independent of $t$, such that $R(t)=R^{0}-\sum_{i=1}^{n} \frac{x_{i}(t)}{y R_{i}}<\alpha<\lambda_{R_{j}}$ for $j<k$. Therefore if $x_{j}(t)>0$ for some $j<k$ then $x_{j}^{\prime}(t)<0$. It follows that if $S\left(t_{0}\right)=\lambda_{S_{k}}$ for some $t_{0}, S^{\prime}\left(t_{0}\right)=$ $-\sum_{i=1}^{n} \frac{x_{i}^{\prime}\left(t_{0}\right)}{y S_{i}}>0$. Consequently, if $S\left(t_{0}\right)=\lambda_{S_{k}}$ then $S(t)>\lambda_{S_{k}}$ for $t>t_{0}$. In other words, if $S(\bar{t})>\lambda_{S_{k}}$ for some $\bar{t} \geq 0$ then this inequality holds for all $t>\bar{t}$. Therefore without loss of generality, we only need to consider the two cases that $S(t)<\lambda_{S_{k}}$ for $t \geq 0$ or $S(t)>\lambda_{S_{k}}$ for $t \geq 0$.

Suppose that $S(t)<\lambda_{S_{k}}$ for all $t \geq 0$. Since the argument above shows that $S^{\prime}(t)>0$ if $S(t)<\lambda_{S_{k}}$, the limit $\bar{S}=\lim _{t \rightarrow \infty} S(t) \leq \lambda_{S_{k}}$ exists. From the argument above it follows that for each $j \neq k, x_{j}^{\prime}(t) \leq \alpha_{j} x_{j}(t)$ where $\alpha_{j}=\max _{0 \leq S \leq \lambda_{S_{k}}}\left\{f_{j}(S(t)\right.$, $R(t))-D\}<0$. This shows $\lim _{t \rightarrow \infty} x_{j}(t)=0$ for $j \neq k$. On the other hand, since $x_{k}^{\prime}(t) \leq 0, \bar{x}_{k}=\lim _{t \rightarrow \infty} x_{k}$ exists. Therefore, $x(t)$ converges and, of course, its limit must be one of the steady states. Since $\bar{S}=S^{0}-\frac{\bar{x}_{k}}{y_{S_{k}}} \leq \lambda_{S_{k}}$, we see that $\bar{x}_{k}>0$ and therefore $x(t)$ converges to $F_{S_{k}}$ since $F_{R_{k}}$ does not exist.

Next we assume that $S(t)>\lambda_{S_{k}}$ for all $t \geq 0$. Suppose that $R(t) \leq \lambda_{R_{k}}$ for some $t$. If $j<k$, and if $\lambda_{R_{j}}<\lambda_{R_{k}}$ then $T_{j k}>T_{k}>C_{k}$, contradicting (i). Thus, $\lambda_{R_{j}}>\lambda_{R_{k}}$ for $j<k$ and so $x_{j}^{\prime}(t)<0$ if $x_{j}(t)>0$ for $j<k$. Lemma 6.6 and condition (ii) implies that if $j>k$ then either $\lambda_{R_{k}}<\lambda_{R_{j}}$ or there is a $\beta>0$ such that $S(t)<\beta<\lambda_{S_{j}}$ so $x_{j}^{\prime}(t)<0$ in either case if $x_{j}(t)>0$. Therefore if for some $t_{1} \geq 0, R\left(t_{1}\right)=\lambda_{R_{k}}$, then $R^{\prime}=-\sum_{i=1}^{n} \frac{x_{i}^{\prime}}{y R_{i}}>0$ at $t_{1}$. It follows that if $R(\bar{t})>\lambda_{R_{k}}$ for some $\bar{t} \geq 0$ then this inequality holds for all $t>\bar{t}$. Thus without loss of generality, we assume that either $R(t)<\lambda_{R_{k}}$ for $t \geq 0$ or $R(t)>\lambda_{R_{k}}$ for $t \geq 0$. Suppose first that $R(t)<\lambda_{R_{k}}$ for all $t \geq 0$. The above argument shows that for each $j \neq k$ there exists $\beta_{j}>0$ such that $x_{j}^{\prime}(t) \leq-\beta_{j} x_{j}(t)$ for $t \geq 0$. Therefore $x_{j}(t) \rightarrow 0$ as $t \rightarrow \infty$

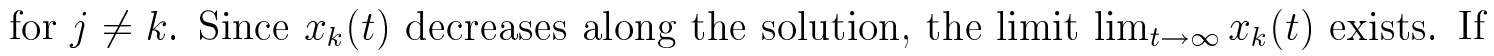


$\lim _{t \rightarrow \infty} x_{k}(t)=0$, then $\left.\lim _{t \rightarrow \infty} R(t)=R^{0}-\lim _{t \rightarrow \infty} \sum_{i=1}^{n} \frac{x_{i}(t)}{y R_{i}}\right)=R^{0}$, a contradiction because of $R(t)<\lambda_{R_{k}}$ for all $t \geq 0$. We therefore must have $\lim _{t \rightarrow \infty} x_{k}(t)=\bar{x}_{k}>0$. Since $x(t)$ converges, it converges to a steady state and so $x(t)$ converges to $F_{S_{k}}$ ( $F_{R_{k}}$ does not exist).

Finally, we consider that $S(t)>\lambda_{S_{k}}$ and $R(t)>\lambda_{R_{k}}$ for $t \geq 0$. In this case, since $x_{k}$ increases along $x(t), \lim _{t \rightarrow \infty} x_{k}(t)$ exists and $\lim _{t \rightarrow \infty} x_{k}(t)>0$. By Lemma 6.4, $\lim _{t \rightarrow \infty}\left[f_{k}(S(t), R(t))-D\right]=0$. Suppose that for some $i_{0} \neq k, \limsup _{t \rightarrow \infty} x_{i_{0}}(t)>$ 0. We derive a contradiction as follows. Lemma 6.3 and Lemma 6.4 show that there exists a sequence $t_{m} \rightarrow \infty$ such that $\lim _{m \rightarrow \infty}\left[f_{i_{0}}\left(S\left(t_{m}\right), R\left(t_{m}\right)\right)-D\right]=0$ and there exists a subsequence of $\left\{t_{m}\right\}$, still denoted by $t_{m}$, such that $\lim _{m \rightarrow \infty} S\left(t_{m}\right)=$ $\bar{S}, \lim _{m \rightarrow \infty} R\left(t_{m}\right)=\bar{R}$, and $\lim _{m \rightarrow \infty} x_{i}\left(t_{m}\right)=\bar{x}_{i}$ exist. It follows that $(\bar{S}, \bar{R})=$ $\left(\lambda_{S_{i_{0}}}, \lambda_{R_{k}}\right)$ and $\lambda_{R_{i_{0}}}<\lambda_{R_{k}}$ if $k<i_{0}$ or $(\bar{S}, \bar{R})=\left(\lambda_{S_{k}}, \lambda_{R_{i_{0}}}\right)$ and $\lambda_{R_{k}}<\lambda_{R_{i_{0}}}$ if $k>i_{0}$. This is because $(\bar{S}, \bar{R})$ must belong to both isoclines $f_{k}-D=0$ and $f_{i_{0}}-D=0$ in the $S-R$ plane and they intersect only if the one with larger $\lambda_{S}$ has smaller $\lambda_{R}$. Keeping in mind that $\bar{S}=S^{0}-\sum_{i=1}^{n} \frac{\bar{x}_{i}}{y_{S_{i}}}$ and similarly for $\bar{R}$, if $\bar{S}=\lambda_{S_{k}}$ then $\bar{R}<\lambda_{R_{j}}$ for $j<k$ by Lemma 6.6 but this contradicts that $\bar{R}=\lambda_{R_{i_{0}}}$ and $i_{0}<k$. If $\bar{R}=\lambda_{R_{k}}$ then $\bar{S}<\lambda_{S_{j}}$ for $k<j$ by Lemma 6.6. But this contradicts that $\bar{S}=\lambda_{S_{i_{0}}}$ and $i_{0}>k$. So we must have $\lim _{t \rightarrow \infty} x_{j}(t)=0$ for $j \neq k$. Since $x_{k}(t)$ is increasing along the solution $x(t)$ and hence $\lim _{t \rightarrow \infty} x_{k}(t)$ exists, condition $T_{k}>C_{k}$ shows that $x(t)$ converges to $F_{S_{k}}$.

The proof of Lemma 6.3 is similar, so we shall omit it.

Proof of Theorem 4.3. It is easily verified that conditions of Theorem4.3 implies that $E_{S_{k}}$ is locally asymptotically stable for(2.1). It suffices to show that $E_{S_{k}}$ belongs to the omega-limit set, $\Omega$, of any solution $x(t)=\left(S(t), R(t), x_{1}(t), \ldots, x_{n}(t)\right)$ of $(2.1)$ with $x_{k}(0)>0$.

Suppose $\lim \sup _{t \rightarrow \infty} x_{k}(t)>0$. Then by Lemma $6.2, E_{S_{k}}$ belongs to the closure 
of $\{x(t): t \geq 0\}$ and hence belongs to $\Omega$. If we assume that $\limsup _{t \rightarrow \infty} x_{k}(t)=0$ and hence $\lim _{t \rightarrow \infty} x_{k}(t)=0$, we derive a contradiction as follows. In this case, $\lim _{t \rightarrow \infty} x_{k}^{\prime}(t)=0$ and $\liminf _{t \rightarrow \infty} S(t) \leq \lambda_{S_{k}}$ or $\liminf _{t \rightarrow \infty} R(t) \leq \lambda_{R_{k}}\left(\right.$ or $x_{k}(t)$ increases to a positive limit). Assume $\liminf _{t \rightarrow \infty} S(t) \leq \lambda_{S_{k}}$. Then there exists a sequence $t_{m} \rightarrow \infty$ such that $\lim _{m \rightarrow \infty} S^{\prime}\left(t_{m}\right)=0, \lim _{m \rightarrow \infty} S\left(t_{m}\right)=\bar{S}, \lim _{m \rightarrow \infty} R\left(t_{m}\right)=$ $\bar{R}, \lim _{m \rightarrow \infty} x_{i}\left(t_{m}\right)=\bar{x}_{i}$, and $\bar{S} \leq \lambda_{S_{k}}$. As $\bar{S}=S^{0}-\sum_{i=1}^{n} \frac{\bar{x}_{i}}{y_{S_{i}}}$ and $\bar{R}=R^{0}-\sum_{i=1}^{n} \frac{\bar{x}_{i}}{y_{R_{i}}}$, it follows from condition (i) and Lemma 6.6 that $\bar{R}<\lambda_{R_{j}}$ for $j<k$. Choose $\varepsilon$ sufficiently small and let $c_{i}=\max _{0 \leq S \leq \bar{S}+\varepsilon, 0 \leq R \leq \bar{R}+\varepsilon}\left[f_{i}(S, R)-D\right]$. Because $\bar{S} \leq \lambda_{S_{k}}$ and $\bar{R}<\lambda_{R_{j}}$ for $j<k$, it follows that $c_{i}<0$ and for large $m, x_{i}^{\prime}\left(t_{m}\right) \leq c_{i} x_{i}\left(t_{m}\right) \leq 0$ for $i \neq k$. Let $z(t)=S(t)+\sum_{i=1}^{n} \frac{x_{i}(t)}{y S_{i}}$. Then $\lim _{t \rightarrow \infty} z(t)=S^{0}$ and hence $\lim _{t \rightarrow \infty} z^{\prime}(t)=0$. In particular, $\lim _{m \rightarrow \infty} z^{\prime}\left(t_{m}\right)=0$. It follows that $\lim _{m \rightarrow \infty} x_{i}^{\prime}\left(t_{m}\right)=0$ and therefore $\lim _{m \rightarrow \infty} x_{i}\left(t_{m}\right)=0$ for $i \neq k$. Thus $\lim _{m \rightarrow \infty} x_{i}\left(t_{m}\right)=0$ for all $1 \leq i \leq n$, which implies that $\lim _{m \rightarrow \infty} S\left(t_{m}\right)=S^{0}>\lambda_{S_{k}}$, a contradiction. If $\liminf _{t \rightarrow \infty} R(t) \leq \lambda_{R_{k}}$, similar argument and condition (ii) can be used to obtain a contradiction too.

The proof of Theorem 4.4 is similar, so we shall omit it.

In order to prove Theorem 4.5, we need the global stability result of the coexistence steady state when there are only two species in the chemostat. In the case of two species, the proofs given in Hsu et. al [15] are valid for monotone increasing nutrient uptake functions. In fact, the proof of Theorem 5.6 in [15] is valid for functions satisfying our assumptions, giving the following conclusion.

Lemma 6.7 Let $n=2$. If $\lambda_{R_{2}}<\lambda_{R_{1}}<R^{0}$ and $C_{1}>T_{12}>C_{2}$, then $E_{12}$ attracts all solutions of (2.1) with $x_{i}(0)>0, i=1,2$.

Proof of Theorem 4.5. Let $\left(S(t), R(t), x_{1}(t), \ldots, x_{n}(t)\right)$ be a solution of $(2.1)$ with $x_{k}(0)>0$ and $x_{l}(0)>0$. We first assume $n>l$ and show $x_{n}(t) \rightarrow 0$ as $t \rightarrow \infty$. Suppose that $\lim _{t \rightarrow \infty} x_{n}(t)$ exists and $\lim _{t \rightarrow \infty} x_{n}(t)>0$. By Lemma 6.4, 
$\lim _{t \rightarrow \infty} x_{n}(t)\left[f_{n}(S(t), R(t))-D\right]=0$ and hence $\lim _{t \rightarrow \infty}\left[f_{n}(S(t), R(t))-D\right]=0$. If $\lim _{m \rightarrow \infty} R\left(t_{m}\right)=\lambda_{R_{n}}$ for a sequence $t_{m} \rightarrow \infty$, we may as well assume that $\lim _{m \rightarrow \infty} S\left(t_{m}\right)=\bar{S}$ and $\lim _{m \rightarrow \infty} x_{i}\left(t_{m}\right)=\bar{x}_{i}$ and, using $h=j=n$ in condition (ii) and Lemma 6.6, we find that $\bar{S}<\lambda_{S_{n}}$, a contradiction. It follows that $\lim _{t \rightarrow \infty} S(t)$ exists, $\lim _{t \rightarrow \infty} S(t)=\lambda_{S_{n}}$ and $\liminf _{t \rightarrow \infty} R(t) \geq \lambda_{R_{n}}$. On the other hand, by condition (ii) with $j=n, h=k$, Lemma 6.6 and a similar argument, $\lim _{t \rightarrow \infty} S(t)=\lambda_{S_{n}}$ implies that $\liminf _{t \rightarrow \infty} R(t) \geq \lambda_{R_{k}}>\lambda_{R_{l}}$. This leads to the conclusion that $x_{l}(t)$ increases without bound, a contradiction. Therefore, if $\lim _{t \rightarrow \infty} x_{n}(t)$ exists, we must have $\lim _{t \rightarrow \infty} x_{n}(t)=0$.

If we suppose that $x_{n}(t)$ does not converge then its limit superior exceeds its limit inferior. Lemma 6.5 shows that there are two sequences $t_{m} \rightarrow \infty$ and $\bar{t}_{m} \rightarrow \infty$ such that $x_{n}^{\prime}\left(t_{m}\right)=0, x_{n}^{\prime}\left(\bar{t}_{m}\right)=0, \lim _{m \rightarrow \infty} x_{n}\left(t_{m}\right)=\gamma$ and $\lim _{m \rightarrow \infty} x_{n}\left(t_{m}\right)=\sigma$, where $\gamma=\limsup _{t \rightarrow \infty} x_{n}(t)$ and $\sigma=\liminf _{t \rightarrow \infty} x_{n}(t)$. We can assume that the local maxima and local minima of $x_{n}(t)$ are attained at $t_{m}$ and $\bar{t}_{m}$ respectively. Clearly, $f_{n}\left(S\left(t_{m}\right), R\left(t_{m}\right)\right)-D=0$ and $f_{n}\left(S\left(\bar{t}_{m}\right), R\left(\bar{t}_{m}\right)\right)-D=0$. We may assume that $S\left(\bar{t}_{m}\right) \rightarrow \bar{S}, R\left(\bar{t}_{m}\right) \rightarrow \bar{R}$ and $x_{i}\left(\bar{t}_{m}\right) \rightarrow \bar{x}_{i}$ where $\bar{S}=S^{0}-\sum_{i=1}^{n} \frac{\bar{x}_{i}}{y_{S_{i}}}, \bar{R}=$ $R^{0}-\sum_{i=1}^{n} \frac{\bar{x}_{i}}{y_{R_{i}}}$ and $f_{n}(\bar{S}, \bar{R})-D=0$. If $\bar{R}=\lambda_{R_{n}}$, then arguing as in the previous paragraph we conclude that $\bar{S}<\lambda_{S_{n}}$ which is impossible. Therefore, $\bar{S}=\lambda_{S_{n}}$. But if $\bar{S}=\lambda_{S_{n}}$, then (ii) with $j=h, k=n$ and Lemma 6.6 imply that $\bar{R}>\alpha>\lambda_{R_{i}}$ for $i \geq$ 1 so $R\left(\bar{t}_{m}\right) \geq \alpha>\lambda_{R_{i}}$ holds for each $i$ and all large $m$. It follows that there exists a constant $a_{i}>0$ such that $x_{i}^{\prime}\left(\bar{t}_{m}\right) \geq a_{i} x_{i}\left(\bar{t}_{m}\right)$ for $i \neq n$. Note that $f_{n}\left(S\left(\bar{t}_{m}\right), R\left(\bar{t}_{m}\right)\right)=$ $p_{n}\left(S\left(\bar{t}_{m}\right)\right)$ and hence $x_{n}^{\prime \prime}$ exists at $\bar{t}_{m}$. Since $x_{n}$ attains a local minimum at $\bar{t}_{m}$, we have $x_{n}^{\prime \prime}\left(\bar{t}_{m}\right) \geq 0$. The chain rule shows $S^{\prime}\left(\bar{t}_{m}\right) \geq 0$. Let $z(t)=S(t)+\sum_{i=1}^{n} \frac{x_{i}(t)}{y_{S_{i}}}$. Since $\lim _{t \rightarrow \infty} z(t)=S^{0}, \lim _{t \rightarrow \infty} z^{\prime}(t)=0$ and in particular, $\lim _{m \rightarrow \infty} z^{\prime}\left(\bar{t}_{m}\right)=0$. It follows that $\lim _{t \rightarrow \infty} x_{i}^{\prime}\left(\bar{t}_{m}\right)=0$ and therefore $\lim _{m \rightarrow \infty} x_{i}\left(\bar{t}_{m}\right)=0$ for $i \neq n$. This leads to $S^{0}=\lambda_{S_{n}}+\sigma / y_{S_{n}}$ and thus $\sigma=\left(S^{0}-\lambda_{S_{n}}\right) y_{S_{n}}$. On the other hand, 
since $\lim _{m \rightarrow \infty} z\left(t_{m}\right)=S^{0}, \lim _{m \rightarrow \infty} S\left(t_{m}\right)=\lambda_{S_{n}}, \lim _{m \rightarrow \infty} x_{n}\left(t_{m}\right)=\gamma$, it follows that $S^{0} \geq \lambda_{S_{n}}+\gamma / y_{S_{n}}$ and thus $\gamma \leq\left(S^{0}-\lambda_{S_{n}}\right) y_{S_{n}}$. This shows that $\gamma=\sigma$, a contradiction. We have shown $\lim _{t \rightarrow \infty} x_{n}(t)=0$.

Using induction and similar arguments, one can show $\lim _{t \rightarrow \infty} x_{j}(t)=0$ for all $j>l$. Similarly, one can use condition (iii) to show $\lim _{t \rightarrow \infty} x_{j}(t)=0$ for $j<l$ and $j \neq k$. Here, one starts by showing that $x_{k_{l-2}}(t)$ converges to zero, then $x_{k_{l-3}}(t)$ and so forth. Therefore we must have that $p_{i}=0, i \neq k, l$ for all points $(\bar{S}, \bar{R}, p)$ in the omega limit set $\Omega$ of our solution $(S(t), R(t), x(t)$. Note that condition (i) of Theorem 4.5 and Lemma 6.4 show that $E_{k l}$ is locally asymptotically stable and attracts all solutions of the $x_{k}-x_{l}$ subsystem with positive values of $x_{k}(0)$ and $x_{l}(0)$. It follows that if $\Omega$ contains a point $e=(\bar{S}, \bar{R}, p)$ with $p_{l}>0$ and $p_{k}>0$ then $E_{k l} \in \Omega$ and consequently $\Omega=\left\{E_{k l}\right\}$. Suppose that $\Omega \neq\left\{E_{k l}\right\}$ and point $e=(\bar{S}, \bar{R}, p) \in \Omega$. Then either $p_{k}=0$ or $p_{l}=0$ (as well as $p_{i}=0$ for all $i \neq k, l$ ). But then the invariance of the limit set implies that it must contain $E_{0}, E_{R_{k}}$ or $E_{S_{l}}$. $\Omega=\left\{E_{0}\right\}$ is easily ruled out since $f_{k}-D>0$ and $f_{l}-D>0$ nearby. Thus $\Omega$ must contain $E_{R_{k}}$ or $E_{S_{l}} . \Omega=\left\{E_{R_{k}}\right\}$ is impossible because by (i) $f_{l}-D>0$ at $E_{R_{k}}$ implying that $x_{l}$ grows without bound if the solution remains in a small neighborhood of $E_{R_{k}}$. A similar argument shows that $\Omega=\left\{E_{S_{l}}\right\}$ is also impossible. Therefore, $\Omega$ must contain one of these steady states and other points as well. Then the ButlerMcGehee Lemma implies that if $E_{R_{k}} \in \Omega$ then it contains a point of the unstable manifold of $E_{R_{k}}$ other than $E_{R_{k}}$ and similarly if $E_{S_{l}} \in \Omega$. As the orbit through such a point converges to $E_{k l}$ we have arrived at the contradiction $E_{k l} \in \Omega$. Therefore, we must have $\Omega=\left\{E_{k l}\right\}$.

\subsection{Proofs for the case $n=3$}


The reduced system (2.15) is defined on the subset of $\mathbb{R}_{+}^{3}$ defined by

$$
\Gamma \equiv\left\{x \in \mathbb{R}_{+}^{3}: \sum_{i=1}^{3} \frac{x_{i}}{y_{S_{i}}} \leq S^{0}, \sum_{i=1}^{3} \frac{x_{i}}{y_{R_{i}}} \leq R^{0}\right\} .
$$

It will be convenient to extend it to all of $\mathbb{R}_{+}^{3}$ by replacing $S^{0}-\sum_{i=1}^{3} \frac{x_{i}}{y_{S_{i}}}$ by its positive part $\left[S^{0}-\sum_{i=1}^{3} \frac{x_{i}}{y_{S_{i}}}\right]_{+}$and similarly for the other argument of $f_{i}$. The resulting vector field is globally Lipschitz on $\mathbb{R}_{+}^{3}$.

Note that (2.10) ensures that the reduced system is continuously differentiable in a neighborhood of each of its steady states and that each is hyperbolic. All steady states of the reduced system belong to $\partial \mathbb{R}_{+}^{3} \cap \Gamma$. Recall our notation $E_{i}$ for either $E_{S_{i}}$ or $E_{R_{i}}$, whichever exists. In this section, we will abuse notation and use this notation for the steady states of both the reduced system (2.15) and the original system (2.1).

Theorem 4.6 will follow from the following result and a convergence theorem of [31].

Theorem 6.1 Every solution $x(t)$ of (2.15) with $x_{i}(0)>0,1 \leq i \leq 3$ converges to one of the steady states $E_{i}, E_{i j}$ on the boundary of $\mathbb{R}_{+}^{3}$. There are no heteroclinic cycles of steady states for (2.15).

Proof of Theorem 4.6. The proof follows from Theorem F.1 in [32] and Theorem 6.1 on rewriting (2.1) in the form of a coupled system for the variables $z_{1}=S(t)-\sum_{i} \frac{x_{i}}{y_{S_{i}}}$ and $z_{2}=R(t)-\sum_{i} \frac{x_{i}}{y_{R_{i}}}$ and the $x_{i}$.

In order to prove Theorem 6.1, we require several preliminary results. In each, $\omega=\omega(p)$ is the omega limit set of a point $p$ with $p \in \mathbb{R}_{+}^{3}$ and $p_{i}>0,1 \leq i \leq 3$.

Lemma 6.8 The extended reduced system is a competitive system in the sense that its time-reversal satisfies the Kamke conditions and hence the forward flow of the latter preserves the standard partial order $\leq$ on $\mathbb{R}^{3}$. 
Proof: We must show that the time-reversed system $x_{i}^{\prime}=-\left(f_{i}-D\right) x_{i} \equiv F_{i}$, $1 \leq i \leq 3$, satisfies the Kamke condition [32] : $x, \bar{x} \in \mathbb{R}_{+}^{3}, x \leq \bar{x}$, and $x_{i}=\bar{x}_{i}$ implies that $F_{i}(x) \leq F_{i}(\bar{x})$. We have $S \equiv S^{0}-\sum_{i} \frac{x_{j}}{y_{S_{j}}} \geq S^{0}-\sum_{i} \frac{\overline{x_{j}}}{y_{S_{j}}} \equiv \bar{S}$ so $S_{+} \geq \bar{S}_{+}$and $p_{i}\left(S_{+}\right) \geq p_{i}\left(\bar{S}_{+}\right)$. Similarly, $q_{i}\left(R_{+}\right) \geq q_{i}\left(\bar{R}_{+}\right)$. Thus, $f_{i}\left(S_{+}, R_{+}\right)-D \geq$ $f_{i}\left(\bar{S}_{+}, \bar{R}_{+}\right)-D$ and the desired conclusion follows.

Lemma $6.9 \omega \subset \Gamma$ and $\omega$ contains at least one steady state. It contains no point of the boundary of $\Gamma$ relative to $\mathbb{R}_{+}^{3}$ and $E_{0} \notin \omega$.

Proof: Note that $x_{i}^{\prime} \leq-D x_{i}$ if either $\sum_{i=1}^{3} \frac{x_{i}}{y_{S_{i}}} \geq S^{0}$ or $\sum_{i=1}^{3} \frac{x_{i}}{y_{R_{i}}} \geq R^{0}$. Consequently, $\left[\sum_{i} \frac{x_{i}}{y_{S_{i}}}\right]^{\prime} \leq-D \sum_{i} \frac{x_{i}}{y_{S_{i}}}$, and similarly with $R$ replacing $S$. It follows immediately that every solution eventually enters and remains in $\Gamma$ and that $\sum_{i=1}^{3} \frac{x_{i}}{y_{S_{i}}}<S^{0}$ and $\sum_{i=1}^{3} \frac{x_{i}}{y_{R_{i}}}<R^{0}$ on $\omega$. If $y_{i}>0,1 \leq i \leq 3$ for every $y \in \omega$ then by the compactness of $\omega$ we could find $a, b>0$ such that $a<y_{i}<b, 1 \leq i \leq 3$ holds for all $y \in \omega$. By a result of Hirsch [11] (see also Theorem 3.4.1 in [32]), as $\omega$ contains no steady state, it must be a nontrivial periodic orbit. But then by a result of [32] (see Proposition 3.4.3 and note that the $C^{1}$ hypothesis is used only to check for competitiveness and is not essential), there must exist a steady state $e$ with $a \leq e_{i} \leq b, 1 \leq i \leq 3$. This contradiction implies that some point $q \in \omega$ satisfies $q_{i}=0$ for some $i$. As $E_{0}$ is a repeller, $E_{0} \notin \omega$ and so $q_{j}>0$ for some $j$. The invariance and compactness of the limit set imply that $\omega$ contains the omega limit set of $q$ and hence some steady state, as all trajectories on $\partial \mathbb{R}_{+}^{3}$ converge to some steady state by Lemma 6.7.

Lemma $6.10 \omega$ does not contain any steady state $e$ with a one-dimensional stable manifold $W^{s}(e)$.

Proof: There are two cases, $e$ is axial $\left(E_{i}\right)$ or it's planar $\left(E_{i j}\right)$. In either case, $W^{s}(e)$ consists of $e$ and two entire orbits which lie on the axis, if $e$ is axial, or in the 
coordinate plane containing $e$, if $e$ is planar. One of these orbits is a heteroclinic one connecting $e$ to $E_{0}$ and the other connects $e$ to the point at infinity. $\omega=\omega(p)=\{e\}$ is impossible since $p \notin W^{s}(e)$. $e \notin \omega$ because the Butler-McGehee Lemma would imply that $\omega$ contain a point of $W^{s}(e)$ different from $e$. This is impossible as it would imply that $E_{0} \in \omega$ or that the point at infinity belongs to $\omega$.

Lemma 6.11 Let $i, j, k$ be distinct and suppose that $f_{k}-D>0$ at $E_{i}$ and $E_{j}$. Then $f_{k}-D>0$ at $E_{i j}$ if it exists. The analogous result when $f_{k}-D<0$ holds provided $E_{i j}$ is asymptotically stable in the linear approximation in the $x_{i}-x_{j}$ plane.

Proof: If $f_{k}-D>0$ at point $P=\left(S^{*}, R^{*}\right)$ then there exists $\epsilon>0$ such that $f_{k}-D>0$ at all points of $\left\{(S, R): S>S^{*}-\epsilon, R>R^{*}-\epsilon\right\}$. Furthermore, if $f_{k}-D>0$ at points $P$ and $Q=\left(S^{\prime}, R^{\prime}\right)$, then $f_{k}-D>0$ at $T=P \wedge Q=$ $\left(\min \left\{S^{*}, S^{\prime}\right\}, \min \left\{R^{*}, R^{\prime}\right\}\right)$.

It is useful to return to the original variables $S$ and $R$ and to identify the steady states with the corresponding ordered pair $(S, R)$ according to $S=S^{0}-\sum_{i=1}^{3} \frac{x_{i}}{y_{S_{i}}}$ and $R=R^{0}-\sum_{i=1}^{3} \frac{x_{i}}{y_{R_{i}}}$. Assume without loss of generality that $i<j$ (so $\lambda_{S_{i}}<\lambda_{S_{j}}$ ) and that $E_{i j}$ exists, hence $\lambda_{R_{j}}<\lambda_{R_{i}}$. It follows that $E_{i j}=\left(\lambda_{S_{j}}, \lambda_{R_{i}}\right)$. There are two cases depending on whether $E_{i j}$ is asymptotically stable or a saddle point relative to the $x_{i}-x_{j}$ plane.

If $E_{i j}$ is a saddle point in the $x_{i}-x_{j}$ plane then $E_{i}=\left(S_{i}, R_{i}\right)$ where $S_{i}<S_{\lambda_{j}}$ and $R_{i} \geq \lambda_{R_{i}} ; E_{j}=\left(S_{j}, R_{j}\right)$ where $S_{j} \geq \lambda_{S_{j}}$ and $R_{j}<\lambda_{R_{i}}$. Thus, $E_{i} \wedge E_{j}=\left(S_{i}, R_{j}\right)<$ $E_{i j}$. Since $f_{k}-D>0$ at $E_{i}$ and at $E_{j}$ we conclude that $f_{k}-D>0$ at $\left(S_{i}, R_{j}\right)$ and, therefore, also at $E_{i j}$.

In the asymptotically stable case, $E_{i}=\left(S_{i}, \lambda_{R_{i}}\right)$ with $S_{i}>\lambda_{S_{j}}$ and $E_{j}=\left(\lambda_{S_{j}}, R_{j}\right)$ with $R_{j}>\lambda_{R_{i}}$. It follows that $E_{i} \wedge E_{j}=E_{i j}$. As $f_{k}-D>0$ at $E_{i}$ and at $E_{j}$, we conclude from the paragraph above that $f_{k}-D>0$ at $E_{i} \wedge E_{j}=E_{i j}$, so we are done in this case. 
Now suppose that the reverse inequality $f_{k}-D<0$ holds at $E_{i}$ and $E_{j}$ and that $E_{i j}$ exists and is asymptotically stable in the linear approximation for the $x_{i}-x_{j}$ subsystem. The $S-R$ coordinates of the steady states are as in the previous paragraph. Suppose that the assertion were false and that $f_{k}-D \geq 0$ at $E_{i j}$. As we assume hyperbolicity of $E_{i j}$, in fact, $f_{k}-D>0$ at $E_{i j}$. Now, $E_{i}, E_{j} \geq E_{i j}$ so from the first paragraph of the proof, we conclude that $f_{k}-D>0$ at $E_{i}$ and at $E_{j}$, a contradiction.

Lemma 6.12 Let $E_{i}$ and $E_{j}$ be axial steady states in the plane $x_{k}=0$. If both are unstable in the $x_{k}$-direction, i.e., if $f_{k}-D>0$ evaluated at $E_{i}$ and $E_{j}$, then $\omega$ contains no point with $x_{k}=0$.

Proof: By Lemma 6.11, our hypotheses imply that if the planar steady state $E_{i j}$ exists in the $x_{k}=0$ plane, then $f_{k}-D>0$ holds when evaluated at $E_{i j}$ as well. We apply persistence theory [37] to show that $x_{k}>0$ on all points of $\omega$. Indeed, the dynamics of the reduced system restricted to the $x_{k}=0$ face of $\partial \mathbb{R}_{+}^{3}$ are simple. All orbits converge to steady state and there are no heteroclinic cycles. The stable manifolds of each of these steady states (including $E_{0}$ ) is, by our hypotheses, entirely contained in the $x_{k}=0$ plane. An acyclic isolated covering can be made up from the steady states, each of which is a weak repeller for $x_{k}>0$. The latter is obvious for $E_{0}$ and follows for the other steady states by virtue of $f_{k}-D>0$, which holds in a neighborhood of each nontrivial steady state. Therefore, we may conclude from Theorem 4.5 [37], that $x_{k}>0$ on $\omega$.

The idea of a saturated equilibrium [13] will be very useful. A steady state $p$ of (2.15) is saturated if $f_{i}-D \leq 0$ at $p$ if $p_{i}=0$. As $f_{i}-D$ is an eigenvalue of the Jacobian when $p_{i}=0$ and because our assumptions rule out degenerate steady states, a saturated steady state $p$ must have $f_{i}-D<0$ at $p$. Thus, the steady state 
cannot be invaded by the ith species. It is easy to see that $E_{i}$ is saturated if and only if it is asymptotically stable, while $E_{i j}$ is saturated if and only if $f_{k}-D<0$, where $i, j, k$ are distinct. The following is an immediate consequence of [13] (Theorem 1 , sec. 19.4 and Exercise 3).

Lemma 6.13 There exists an odd number of saturated equilibria for (2.15).

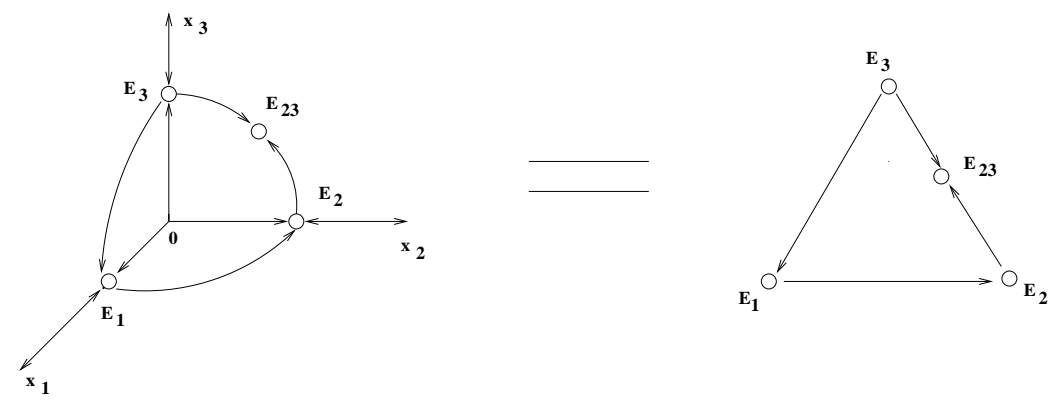

Figure 2: Triangle method of depicting boundary phase diagrams
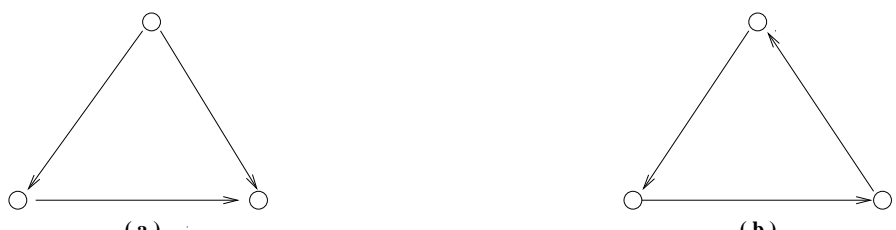

( b )

Figure 3: Case 1: boundary phase diagrams involving 3 steady states.

Proof of Theorem 6.1: The proof is rather long and tedious because there are so many possible phase portraits restricted to $\partial \mathbb{R}_{+}^{3}$ for $(2.15)$ and because our method of proof is dependent on these. It will be useful to give a brief overview of the strategy of the proof. Let $\omega$ denote the limit set of our solution with $x(0)=p$ and $p_{i}>0$ for all $i$. We know that $\omega$ contains a steady state. If $\omega$ contains an asymptotically stable steady then we are done. Lemma 6.10 and Lemma 6.12 identify two impossible scenarios and Lemma 6.13 sets an important constraint. Our 


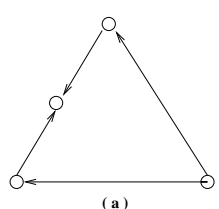

(a)

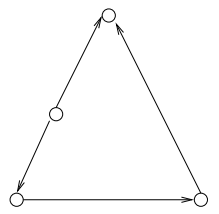

(d)

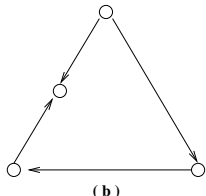

(b)

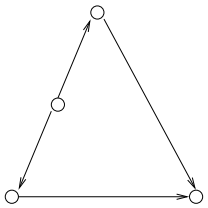

(e)

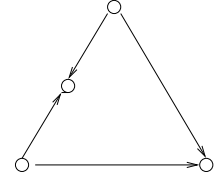

(c)

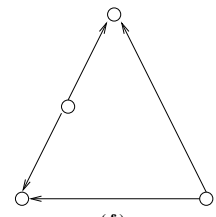

Figure 4: Case 2: boundary phase diagrams involving 4 steady states.
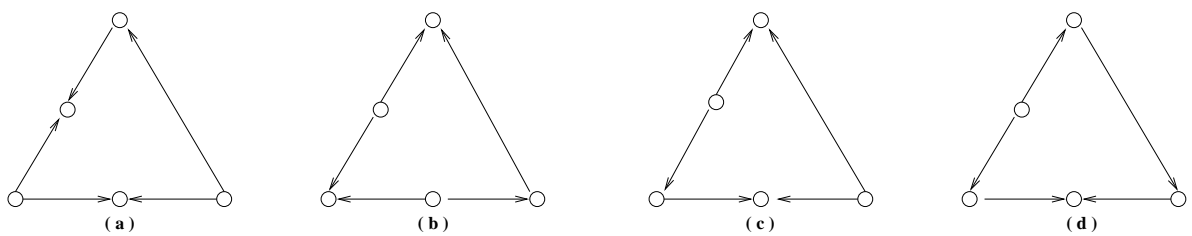

Figure 5: Case 3: boundary phase diagrams involving 5 steady states.

strategy is simple. We use the above-mentioned results to eliminate as many of the steady states as possible from belonging to $\omega$. We use the Butler McGehee lemma to show that $\omega$ must either contain an asymptotically stable steady state or one of the $E_{i j}$. The latter are the only steady states which may be unstable yet attract positive orbits. In pursuing this strategy it is crucial that either the stable manifold $W^{s}$ or the unstable manifold $W^{u}$ of a steady state lies entirely in a coordinate axis or in a coordinate plane of $\mathbb{R}^{3}$ (this is why we only need the "boundary dynamics"). This strategy fails if there is a heteroclinic cycle of steady states.

Obviously, this strategy requires us to consider all potential (some turn out to be impossible) boundary phase portraits. We require a shorthand way of referring to a particular boundary dynamics. As $E_{0}$ is always a source, our shorthand need not include this aspect of the boundary dynamics. The number of steady states 

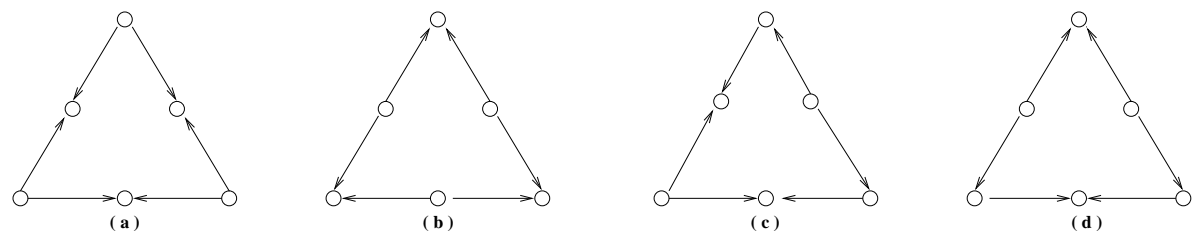

Figure 6: Case 4: boundary phase diagrams involving 6 steady states.

other than $E_{0}$ together with the part of their unstable manifolds belonging to the boundary serve to specify a particular phase portrait. We use triangles to describe boundary phase diagrams with vertices denoting $E_{i}$ 's, points (if any) on lateral sides $E_{i} E_{j}$ denoting $E_{i j}$, and line segments with arrows denoting orbits (belonging to the boundary) that connect steady states. This triangle method is illustrated in Figure 2. We classify the triangle diagrams according to the number of steady states other than $E_{0}$ involved (see Figure 3-Figure 6). In each of these triangle diagrams, the left vertex denotes $E_{1}$, the right vertex denotes $E_{2}$, and the top vertex denotes $E_{3}$, as described in Figure 2. Of course, there are many phase diagrams which can be obtained from a given one by one of the symmetries of the triangle. Imagine the triangle is an equilateral one; there are two 120 rotations of the triangle which lead to equivalent phase diagrams; there are also three symmetries by reflection through one of the altitudes of the triangle which lead to equivalent phase diagrams. Therefore, our catalog of phase diagrams lists only one representative from a set of equivalent phase diagrams obtained by symmetry operations.

We proceed in a case-by-case manner according to the phase diagrams in the Figures 3-Figures 6.

Case 1a: Lemma 6.12 implies that neither $E_{1}$ nor $E_{3}$ belong to $\omega$. Therefore, by Lemma $6.9, E_{2}$ must belong to $\omega$. But $E_{2}$ is locally asymptotically stable so $\omega=\left\{E_{2}\right\}$.

Case 1b: This case, with its heteroclinic cycle $E_{1} \rightarrow E_{2} \rightarrow E_{3} \rightarrow E_{1}$ can't occur 
for there would not exist a saturated equilibrium, contradicting Lemma 6.13.

Case 2a: Lemma 6.10 implies that $E_{2}$ does not belong to $\omega$. If either $E_{1}$ or $E_{3}$ belong to $\omega$ an application of the Butler-McGehee lemma would force $E_{13}$ to belong to $\omega$. By Lemma 6.11 , the latter is locally asymptotically stable so $E_{13}$ coincides with $\omega$.

Case 2b: Lemma 6.12 implies that $E_{3}$ and $E_{2}$ do not belong to $\omega$. Butler-McGehee implies that $E_{1}$ can't belong without forcing $E_{2}$ to belong. Lemma 6.9 and the above arguments imply that $E_{13}$ is the only steady state belonging to $\omega$. It must be saturated by lemma 6.13 . Thus $f_{2}-D<0$ and $E_{13}$ is locally asymptotically stable. In this case, $\omega$ coincides with $E_{13}$.

Case 2c: Lemma 6.12 implies that $x_{2}>0$ on $\omega$. Lemma 6.9 implies that the locally asymptotically stable steady state $E_{2}$ must belong to $\omega$ so it coincides with $\omega$.

Case 2d: If $f_{2}-D<0$ at $E_{13}$ then there would be two saturated steady states, $E_{3}$ being the other. This contradicts Lemma 6.13 so we conclude that $f_{2}-D>0$ at $E_{13}$. Then $E_{13}$ cannot belong to $\omega$ by Lemma 6.10 . The Butler-McGehee lemma implies that $E_{1}$ and $E_{2}$ cannot belong to $\omega$ without also $E_{3}$. Thus, $\omega=\left\{E_{3}\right\}$ in this case since it is asymptotically stable.

Case 2e: same argument as case $4 \mathrm{c} ; \omega=\left\{E_{2}\right\}$.

Case 2f: Lemma 6.10 implies that $E_{2}$ does not belong to $\omega$. As $E_{1}$ and $E_{3}$ are saturated, Lemma 6.13 implies that $E_{13}$ is too so $f_{2}-D<0$ at $E_{13}$. Limit set $\omega$ may coincide with $E_{13}$ provided point $p$ belongs to its two-dimensional stable manifold. However, $E_{13} \in \omega \neq\left\{E_{13}\right\}$ is not possible. Indeed, Butler-McGehee would imply that either $E_{1}$ or $E_{3}$ also belong to $\omega$. But these are both locally asymptotically stable and therefore must coincide with $\omega$ if they belong to $\omega$. Therefore, $\omega$ must coincide with precisely one of the steady states $E_{1}, E_{2}$ or $E_{13}$.

Case 3a: Lemma 6.12 implies that both $x_{3}>0$ and $x_{1}>0$ on $\omega$. Thus, Lemma 6.9 
says that $E_{13}$ is the only steady state that belongs to $\omega$. It must be saturated by Lemma 6.13. It follows that $E_{13}$ is locally asymptotically stable so $\omega=\left\{E_{13}\right\}$.

Case 3b: $E_{2}$ cannot belong to $\omega$ because Butler-McGehee would imply that the asymptotically stable steady state $E_{3}$ is part of $\omega$. Exactly one of $E_{13}, E_{12}$ is saturated as $E_{1}, E_{3}$ are saturated. Suppose that $f_{2}-D<0$ at $E_{13}$ so its stable manifold is two-dimensional containing points $p$ with positive coordinates. Thus, $\omega$ can coincide with it. However, $\left\{E_{13}\right\}$ cannot be a proper subset of $\omega$ because Butler-McGehee would then force one of the locally asymptotically stable steady states $E_{1}$ or $E_{3}$ to make up a proper subset of $\omega$. As $f_{3}-D>0$ at $E_{12}$ it has a one-dimensional stable manifold so it could not belong to $\omega$ by Lemma 6.10. Lemma 6.9 and the above imply that $\omega$ coincides with $E_{1}, E_{3}$ or $E_{13}$. If $f_{2}-D>0$ at $E_{13}$ and $f_{3}-D<0$ at $E_{12}$ then $\omega$ coincides with $E_{1}, E_{3}$ or $E_{12}$.

Case 3c: Lemma 6.10 implies that $E_{2}$ does not belong to $\omega$. Of course, $E_{3}$ is locally asymptotically stable so it may coincide with $\omega$ but not be a proper subset of $\omega$. Either both or neither of $E_{13}, E_{12}$ are saturated by Lemma 6.13. There are thus two subcases:

(i): $f_{2}-D>0$ at $E_{13}$ and $f_{3}-D>0$ at $E_{12}$. Neither planar steady state can coincide with $\omega . E_{13}$ has a one-dimensional stable manifold so cannot belong to $\omega$ by Lemma 6.10 . If $E_{12}$ belonged to $\omega$, then either $E_{2}$ or $E_{0}$ or $E_{1}$ or the point at infinity would also belong. All are immediate contradictions except $E_{1}$. If it belongs, so does $E_{13}$ by Butler-McGehee, a contradiction.

(ii) $f_{2}-D<0$ at $E_{13}$ and $f_{3}-D<0$ at $E_{12} . E_{12}$ is locally asymptotically stable. $E_{1}$ cannot belong to $\omega$ since Butler-McGehee would imply that $E_{12}$ is part of $\omega$. $E_{13}$ may coincide with $\omega$ provided point $p$ belongs to its stable manifold but $\left\{E_{13}\right\}$ cannot be a proper subset of $\omega$ as Butler-McGehee would imply that $E_{1}$ or $E_{3}$ would also belong. Lemma 6.9 implies that $\omega$ coincides with one of the steady states $E_{3}$, 
$E_{12}$ or $E_{13}$.

Case 3d: Lemma 6.12 implies that $x_{2}>0$ on $\omega$. It also implies that $E_{12}$ is locally asymptotically stable. $E_{2}$ cannot belong to $\omega$ since Butler-McGehee would imply that $E_{12}$ is part, but not all, of $\omega$. Thus, $\omega$ coincides with $E_{12}$.

Case 4a: Can't occur. Lemma 6.12 implies that no steady state can belong to $\omega$ and Lemma 6.9 says that some steady state belongs to $\omega$.

Case 4b: Each axial steady state is asymptotically stable in this case. If $f_{2}-D<0$ at $E_{13}$, then it can coincide with $\omega$ if point $p$ belongs to its stable manifold but $E_{13}$ cannot make up a proper subset of $\omega$ because the Butler-McGehee lemma would imply an axial steady state must also make up a part of $\omega$. If $f_{2}-D>0$ at $E_{13}$ then it cannot belong to $\omega$ by Lemma 6.10. Analogous arguments apply to the other two planar steady states. So Lemma 6.9 says $\omega$ contains some nontrivial steady state while arguments above imply that if it contains one of these steady states then it coincides with that steady state. An even number (0 or 2) of the planar steady states are saturated.

In summary, $\omega$ may coincide with any one of the axial steady states or any one of the planar steady states $E_{i j}$ for which $f_{k}-D<0$ at $E_{i j}(i, j, k$ distinct) provided point $p$ belongs to its two-dimensional stable manifold.

Case 4c: Lemma 6.12 implies that $x_{1}>0$ on $\omega$. Lemma 6.10 implies that $E_{1}$ does not belong to $\omega$ so only the two planar steady states $E_{13}$ and $E_{12}$ can belong to $\omega$. Exactly one of these is saturated, which implies it is also asymptotically stable. Two subcases arise.

(i): $f_{3}-D>0$ at $E_{12}$ and $f_{2}-D<0$ at $E_{13}$.

Only $E_{13}$, which is locally asymptotically stable, can belong to $\omega$ and Lemma 6.9 assures that it does belong. Observe that even if there is a positive heteroclinic orbit issuing from $E_{12}$, no heteroclinic cycle of steady states is possible. 
(ii): $f_{3}-D<0$ at $E_{12}$ and $f_{2}-D>0$ at $E_{13}$.

This is the symmetrical case to (ii). $\omega$ coincides with $E_{12}$ and there is no heteroclinic cycle of steady states.

Case 4d: Lemma 6.12 implies that $E_{12}$ is locally asymptotically stable; so is $E_{3}$. Clearly, $E_{1}$ and $E_{2}$ cannot belong to $\omega$ without also $E_{12}$ being part of $\omega$ by ButlerMcGehee, a contradiction. Exactly one of $E_{13}, E_{23}$ are saturated. Again, two subcases arise.

(i): $f_{2}-D>0$ at $E_{13}, f_{1}-D<0$ at $E_{23}$.

$E_{13}$ can't belong to $\omega$ by Lemma $6.10 . E_{23}$ can coincide with $\omega$ if point $p$ belongs to its stable manifold. However, it cannot make up a proper subset of $\omega$ by ButlerMcGehee. So $\omega$ coincides with $E_{3}$ or $E_{12}$ for points not on the stable manifold of $E_{23}$. Although a positive heteroclinic orbit issues from $E_{13}$, no heteroclinic cycle of steady states is possible.

(ii): $f_{2}-D<0$ at $E_{13}, f_{1}-D>0$ at $E_{23}$.

Symmetric to case (ii); $\omega$ coincides with $E_{3}$ or $E_{12}$ for points not on the stable manifold of $E_{13}$. 


\section{Appendix 1.}

In this appendix, we present analysis for local stability of steady states of system (2.1). Since the reduced system (2.19) is more compact, the local stability analysis for its steady states is much easier. On the other hand, the local stability of a steady state of (2.1) is the same as that of the corresponding steady state of (2.19). We shall proceed local stability analysis for the steady states of system (2.19). We investigate the local stability of these steady states by finding the eigenvalues of the associated Jacobian matrices. The Jacobian matrix of (2.19) takes the form

$$
J=\left[\begin{array}{llll}
f_{1}-D+x_{1} \frac{\partial f_{1}}{\partial x_{1}} & x_{1} \frac{\partial f_{1}}{\partial x_{2}} & \cdots & x_{1} \frac{\partial f_{1}}{\partial x_{n}} \\
x_{2} \frac{\partial f_{1}}{\partial x_{2}} & f_{2}-D+x_{2} \frac{\partial f_{2}}{\partial x_{2}} & \cdots & x_{2} \frac{\partial f_{1}}{\partial x_{n}} \\
\vdots & \vdots & \ddots & \vdots \\
x_{n} \frac{\partial f_{n}}{\partial x_{1}} & x_{n} \frac{\partial f_{n}}{\partial x_{2}} & \cdots & f_{n}-D+\frac{x_{n} \partial f_{n}}{\partial x_{n}}
\end{array}\right]
$$

where

$$
f_{i}=f_{i}(S, R), i=1,2, \ldots, n, \quad S=S^{0}-\sum_{i=1}^{n} \frac{x_{i}}{y_{S_{i}}} \quad \text { and } \quad R=R^{0}-\sum_{i=1}^{n} \frac{x_{i}}{y_{R_{i}}} .
$$

At $F_{0}$,

$$
J\left(F_{0}\right)=\left[\begin{array}{llll}
f_{1}\left(S^{0}, R^{0}\right)-D & 0 & \cdots & 0 \\
0 & f_{2}\left(S^{0}, R^{0}\right)-D & \cdots & 0 \\
\vdots & \vdots & \ddots & \vdots \\
0 & 0 & \cdots & f_{n}\left(S^{0}, R^{0}\right)-D
\end{array}\right]
$$

The eigenvalues lie on the diagonal. They are all negative if and only if $f_{i}\left(S^{0}, R^{0}\right)-$ $D<0$ for all $i$, that is either $\lambda_{S_{i}}<S^{0}$ or $\lambda_{R_{i}}<R^{0}$ for all $i$.

When $F_{S_{k}}$ exists, the Jacobian matrix at $F_{S_{k}}$ is $J\left(F_{S_{k}}\right)=$

$$
\left[\begin{array}{llllll}
f_{1}-D & 0 & \cdots & 0 & \cdots & 0 \\
0 & f_{2}-D & \cdots & 0 & \cdots & 0 \\
\vdots & \vdots & \ddots & \vdots & \ddots & 0 \\
\bar{x}_{k} \frac{\partial f_{k}}{\partial x_{1}} & \bar{x}_{k} \frac{\partial f_{k}}{\partial x_{2}} & \cdots & f_{k}-D+\bar{x}_{k} \frac{\partial f_{k}}{\partial x_{k}} & \cdots & \bar{x}_{k} \frac{\partial f_{k}}{\partial x_{n}} \\
\vdots & \vdots & \ddots & \vdots & \ddots & 0 \\
0 & 0 & \cdots & 0 & \cdots & f_{n}-D
\end{array}\right] .
$$


Where $f_{i}=f_{i}\left(\lambda_{S_{k}}, r_{k}\right)$ for $i=1,2, \ldots, n$ and $r_{k}=R^{0}-\frac{\bar{x}_{k}}{y_{R_{k}}}$. Note that when $F_{S_{k}}$ exists, $f_{k}\left(\lambda_{S_{k}}, r_{k}\right)-D$ is zero and $\frac{\partial f_{k}\left(\lambda_{S_{k}}, r_{k}\right)}{\partial x_{k}}<0$. So, The eigenvalues lie on the diagonal. They are all negative if and only if

$$
f_{i}\left(S_{R_{k}}, r_{k}\right)-D<0
$$

for all $i \neq k$. Due to (2.15), (7.4) holds for $i>k$. If $i<k$, then (7.4) holds if and only if $r_{k}<\lambda_{R_{i}}$ or $T_{i k}<C_{k}$.

When $F_{R_{k}}$ exists, the Jacobian matrix at $F_{R_{k}}$ is the same as that at $F_{S_{k}}$ except $f_{i}$ being replaced by $f_{i}\left(s_{k}, \lambda_{R_{k}}\right)$ where $s_{k}=S^{0}-\bar{x}_{k} / y_{S_{k}}, i=1,2, \ldots, n$. The eigenvalues are all negative if and only if

$$
f_{i}\left(s_{k}, \lambda_{R_{k}}\right)-D<0
$$

for all $i \neq k .(7.5)$ is equivalent to

$$
s_{k}<\lambda_{S_{i}} \text { or } \lambda_{R_{k}}<\lambda_{R_{i}}
$$

or

$$
T_{k i}>C_{k} \text { or } \lambda_{R_{k}}<\lambda_{R_{i}}
$$

for all $i \neq k$.

When $F_{k l}$ exists, the Jacobian matrix at $F_{k l}$ is $J\left(F_{S_{k}}\right)=$

$$
\left[\begin{array}{llllllll}
f_{1}-D & 0 & \cdots & 0 & \cdots & 0 & \cdots & 0 \\
0 & f_{2}-D & \cdots & 0 & \cdots & 0 & \cdots & 0 \\
\vdots & \vdots & \ddots & \vdots & \ddots & \vdots & \ddots & \vdots \\
\bar{x}_{k} \frac{\partial f_{k}}{\partial x_{1}} & \bar{x}_{k} \frac{\partial f_{k}}{\partial x_{2}} & \cdots & f_{k}-D+\bar{x}_{k} \frac{\partial f_{k}}{\partial x_{k}} & \cdots & \bar{x}_{k} \frac{\partial f_{k}}{\partial x_{l}} & \cdots & \bar{x}_{k} \frac{\partial f_{k}}{\partial x_{n}} \\
\vdots & \vdots & \ddots & \vdots & \ddots & \vdots & \ddots & \vdots \\
\bar{x}_{l} \frac{\partial f_{l}}{\partial x_{1}} & \bar{x}_{l} \frac{\partial f_{l}}{\partial x_{2}} & \cdots & \bar{x}_{l} \frac{\partial f_{l}}{\partial x_{k}} & \cdots & f_{l}-D+\bar{x}_{l} \frac{\partial f_{l}}{\partial x_{l}} & \cdots & \bar{x}_{l} \frac{\partial f_{l}}{\partial x_{n}} \\
\vdots & \vdots & \ddots & \vdots & \ddots & \vdots & \ddots & \vdots \\
0 & 0 & \cdots & 0 & \cdots & 0 & \cdots & f_{n}-D
\end{array}\right] .
$$


where $f_{i}=f_{i}\left(\lambda_{S_{l}}, \lambda_{R_{k}}\right)$. Note that when $F_{k l}$ exists, $f_{k}-D=f_{l}-D=0$. The eigenvalues of matrix (7.6) consists of

$$
f_{i}\left(\lambda_{S_{l}}, \lambda_{R_{k}}\right)-D, \quad \text { for } i \neq k, l
$$

and the eigenvalues of

$$
\left[\begin{array}{ll}
\bar{x}_{k} \frac{\partial f_{k}}{\partial x_{k}} & \bar{x}_{k} \frac{\partial f_{k}}{\partial x_{l}} \\
\bar{x}_{l} \frac{\partial f_{l}}{\partial x_{k}} & \bar{x}_{l} \frac{\partial f_{l}}{\partial x_{l}}
\end{array}\right] .
$$

Note that at $F_{k l}, \frac{\partial f_{k}}{\partial x_{k}}<0$ and $\frac{\partial f_{k}}{\partial x_{k}}<0$. It follows that the trace of (7.7) is negative and therefore all eigenvalues of (7.7) have negative real parts if and only if

$$
\bar{x}_{k} \bar{x}_{l}\left(\frac{\partial f_{k}}{\partial x_{k}} \frac{\partial f_{l}}{\partial x_{l}}-\frac{\partial f_{k}}{\partial x_{l}} \frac{\partial f_{l}}{\partial x_{k}}\right)>0
$$

which is equivalent to

$$
C_{k}>C_{l}
$$

On the other hand, due to $(2.15), f_{i}\left(\lambda_{S_{l}}, \lambda_{R_{k}}\right)-D<0$ holds for $i>l$. For $i<l$, it holds if and only if $\lambda_{R_{k}}<\lambda_{R_{i}}$. Therefore $F_{k l}$ is locally asymptotically stable if and only if $C_{k}>C_{l}$ and $\lambda_{R_{k}}<\lambda_{R_{i}}$ for $i<l$ and $i \neq k$. 


\section{References}

[1] R. A. Armstrong and R. McGehee, Competitive exclusion, Amer. Natur., 115 (1980), pp. 151-170.

[2] F. G. Bader, Analysis of double-substrate limited growth, Biotechnol. Bioengrg., 20 (1978), pp. 183-202.

[3] M. M. Ballyk and G. S. K. Wolkowicz, An examination of the thresholds of enrichment: a resouce-based growth model, J. Math. Biol., 33 (1995), pp. 435457.

[4] M. M. Ballyk and G. S. K. Wolkowicz, Exploitative competition in the chemostat for two perfectly substitutable resources, Math. Biosci., 118 (1993), 127180.

[5] G. J. Butler and G. S. K. Wolkowicz, A mathematical model of the chemostat with a general class of functions describing nutrient uptake, SIAM J. Appl. Math., 45 (1985), pp. 138-151.

[6] G. J. Butler and G. S. K. Wolkowicz, Exploitative competition in a chemostat for two complementary, and possible inhibitory, resources, Math. Biosci., 83 (1987), pp. 1-48.

[7] J. C. Gottschal and T. F. Thingstad, Mathematical description of competition between two and three bacterial species under dual substrate limitation in a chemostat: A comparison and experimental data, Biotechnol. Bioengrg., 24 (1982), pp. 1403-1418.

[8] J.P. Grover, Resource Competition, Population and Community Biology Series, 19, Chapman\&Hall, New York, 1997. 
[9] S. R. Hansen and S. P. Hubbell, Single-nutrient microbial competition: Qualitative agreement between experimental and theoretically forecast outcomes, Science, 213 (1981), pp. 927-979.

[10] W.M. Hirsch, H. Hanisch, J.P. Gabriel, Differential equation models for some parasitic infections; methods for the study of asymptotic behavior, Comm. Pure Appl. Math.,38 (1985), pp 733-753.

[11] M.W.Hirsch, Systems of differential equations which are competitive or cooperative. I: limit sets, SIAM J.Appl. Math. 13 (1982), pp. 167-179.

[12] C. S. Holling, The functional response of predators to prey density and its role in mimicry and population regulation, Mem. Entomol. Soc. Canada, 45 (1965), pp. 3-60.

[13] J. Hofbauer and K. Sigmund, The Theory of Evolution and Dynamical Systems, London Math. Soc. Student Texts 7, Cambridge University Press, Cambridge, 1988.

[14] S. B. Hsu, Limiting behavior for competing species, SIAM J. Appl. Math., 34 (1978), pp. 760-763.

[15] S. B. Hsu, K. S. Cheng, and S. P. Hubbell, Exploitative competition of microorganism for two complementary nutrients in continuous culture, SIAM J. Appl. Math., 41 (1981), pp. 422-444.

[16] S. B. Hsu, S. Hubbell and P. Waltman, A mathematical theory of single-nutrient competition in continuous cultures of micro-organisms, SIAM J. Appl. Math., 32 (1977), pp. 366-383. 
[17] J. Huisman and F.J. Weissing, Biodiversity of plankton by species oscillations and chaos, Nature ,vol 402, 25 Nov. 1999, pp.407-410.

[18] J. A. Leon and D. B. Tumpson, Competition between two species for two complementary or substitutable resources, J. Theor. Biol., 50 (1975), pp. 185-201.

[19] A.J. Lotka, "Elements of Physical Biology", Williams and Wilkins, Baltimore, 1925.

[20] B. Li, Global asymptotic behaviour of the chemostat; general response functions and different removal rates, SIAM J. Appl. Math., (1999), pp. 411-422.

[21] B. Li, Analysis of Chemostat-Related Models With Distinct Removal Rates, Ph.D thesis, Arizona State University, 1998.

[22] B. Li, G. K. Wolkowicz, and Y. Kuang, Global asymptotic behaviour of a chemostat model with two perfectly complementary resouces and distributed delay, SIAM J. Appl. Math. (in press).

[23] R.M. May and W.J.Leonard, Nonlinear aspects of competition between three species, SIAM J. Appl. Math. 29, 1975, 243-253.

[24] A. Novick and L. Sziliard, Description of the chemostat, Science, 112 (1950), pp. $715-716$.

[25] O. M. Phillips, The equilibrium and stability of simple marine biological systems I. Primary nutrient consumers, Amer. Natur., 107 (1973), pp. 73-93.

[26] D. Rapport, An optimization model of food selection, Amer. Natur. 105(1971), pp. $575-587$.

[27] L. A. Real, The kinetics of functional response, Amer. Natur., 111 (1977), pp. 287-300. 
[28] D. N. Ryder and C. G. Sinclair, Model for the growth of aerobic microorganisms under oxygen limiting conditions, Biotechnol. Bioengrg., 14 (1972), pp. 787-798.

[29] U. Sommer , Competition and Coexistence, Nature, vol 402, 25 Nov. 1999, pp.366-367

[30] R. N. Skyes, Indentification of the limiting nutrient and specific growth rate, J. Water Pollut. Control Fed., 45(1973), pp. 888-895.

[31] H.L. Smith and P. Waltman, "The Theory of the Chemostat," Cambridge University Press, 1995.

[32] H. L. Smith, "Monotone Dynamical Systems, An Introduction to the Theory of Competitive and Cooperative Systems", Mathematical Surveys and Monographs 41, Amer. Math. Soc., Providence, RI, 1995.

[33] H.L.Smith, Periodic orbits of competitive and cooperative systems, J. Diff. Eqns. 65 (1986),pp. 361-373.

[34] D. Tilman and S. S. Kolham, Phosphate and silicate growth and uptake kinetics of the diatoms, Aterionella formosa and Cyclotella meneghiniana, J. Phycol., 12 (1976), pp. 375-383.

[35] D. Tilman, Resource competition between planktonic algae: An experimental and theoretical approach, Ecology, 58 (1977), pp. 338-348.

[36] D. Tilman, Resource competition and Community Structure, Princeton U. P., Princeton, N.J., 1982.

[37] H. R. Thieme, Persistence under relaxed point-dissipativity (with an application to an application to an epidemic model),. SIAM J. Math. Anal, 24 (1993), pp. 407-435. 
[38] V. Volterra, Variations and fluctuations of the number of individuals in animal species living together, J. Conserv. (Conserv. Int. Explor. Mer), 3 (1928), pp. $3-51$.

[39] P. Waltman, S. P. Hubbell, and S. B. Hsu, Theoretical and experimental investigation of microbial competition in continuous culture, in Modeling and Differential Equations, T. Burton, ED., Marcel, Dekker, New York, pp. 105$172,1980$.

[40] G. S. K. Wolkowicz and Z. Lu, Global dynamics of a mathematical model of competition in the chemostat: general response function and differential death rates, SIAM J. Appl. Math., 52 (1992), pp. 222-233.

[41] G. S. K. Wolkowicz and H. Xia, Global asymptotic behavior of a chemostat model with discrete delays, SIAM J. Appl. Math., 57(1997), pp. 1281-1310.

[42] X.-Q. Zhao, Uniform persistence and periodic coexistence states in infinitedimensional periodic semiflows with applications, Canadian Appl. Math. Quart., 3 (1995), 473-495. 


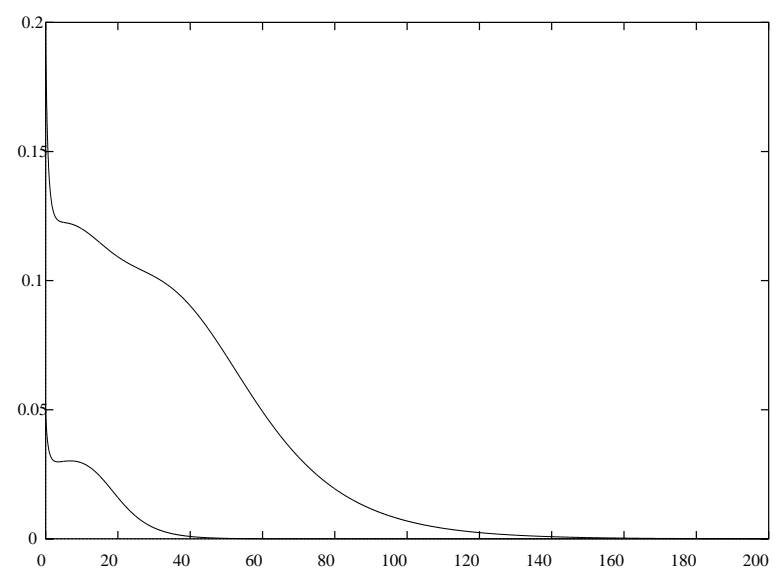

Figure 7: $\left(S(0), R(0), x_{1}(0), x_{2}(0), x_{3}(0), x_{4}(0)\right)=(0.1,0.15,0.2,0.01,0.02,0.05)$. The top curve depicts $x_{1}(t)$, and the bottom one depicts $x_{4}(t)$. Clearly, both $x_{1}(t)$ and $x_{4}(t)$ approach zero. 


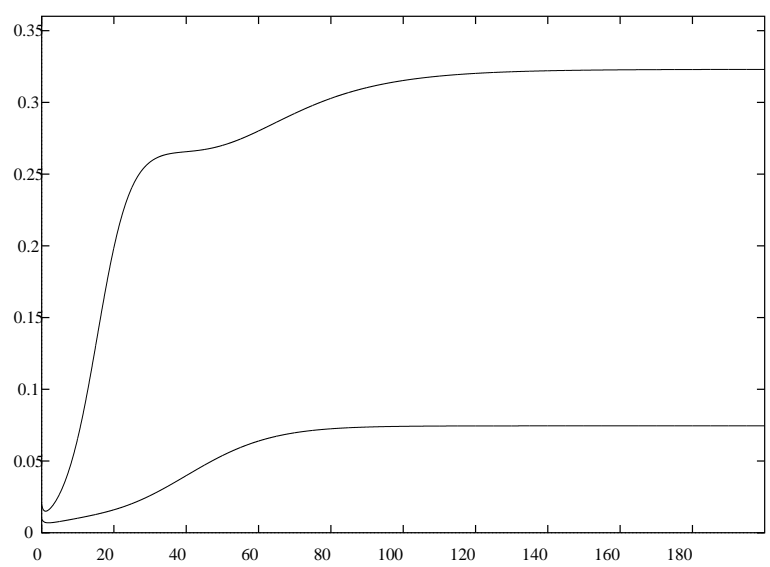

Figure 8: $\left(S(0), R(0), x_{1}(0), x_{2}(0), x_{3}(0), x_{4}(0)\right)=(0.1,0.15,0.2,0.01,0.02,0.05)$. The top curve depicts $x_{2}(t)$, and the bottom one depicts $x_{3}(t)$. Clearly, both $x_{2}(t)$ and $x_{3}(t)$ approach positive constants.

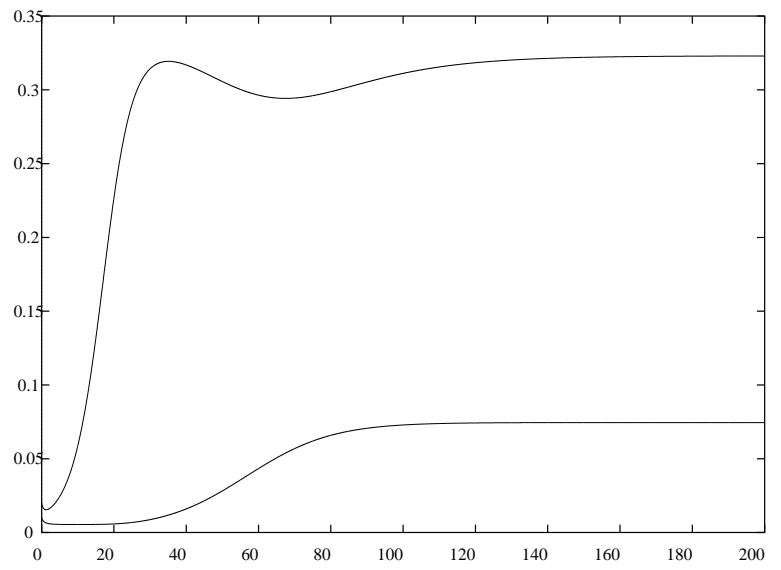

Figure 9: $\left(S(0), R(0), x_{1}(0), x_{2}(0), x_{3}(0), x_{4}(0), x_{5}(0)\right)=(0.16,0.12,0.35,0.01,0.02,0.03$, 0.04). The top curve depicts $x_{2}(t)$, and the bottom one depicts $x_{3}(t)$. Clearly, both $x_{2}(t)$ and $x_{3}(t)$ approach positive constants. 


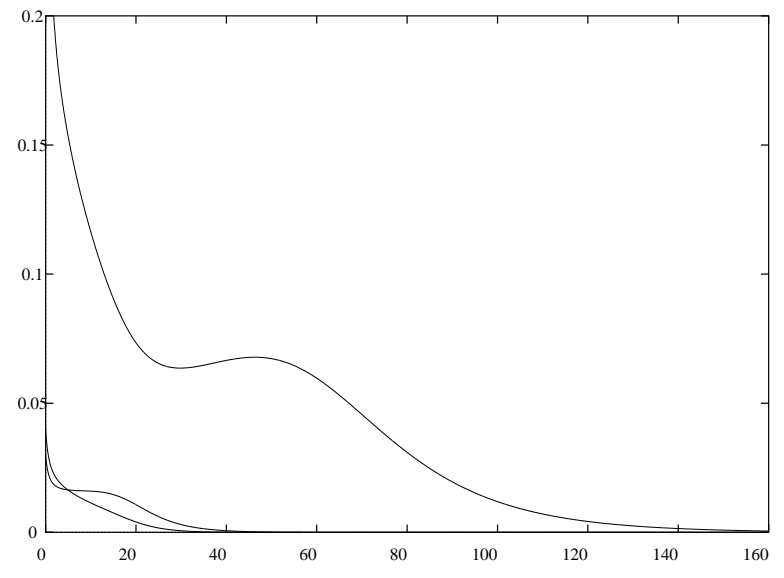

Figure 10: $\left(S(0), R(0), x_{1}(0), x_{2}(0), x_{3}(0), x_{4}(0), x_{5}(0)\right)=(0.16,0.12,0.35,0.01,0.02$, $0.03,0.04)$. The top curve depicts $x_{1}(t)$, the middle curve depicts $x_{4}(t)$, and the bottom one depicts $x_{5}(t)$. Clearly, all $x_{1}(t), x_{4}(t)$, and $x_{5}(t)$ approach zero.

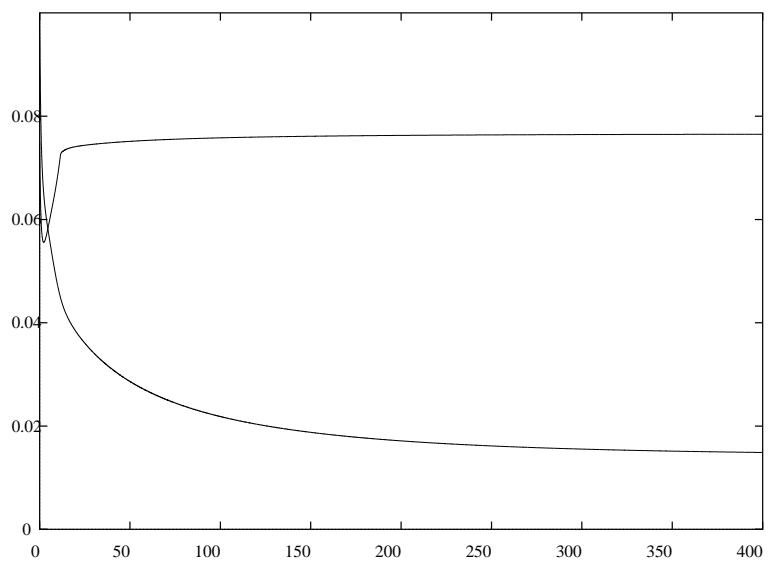

Figure 11: $\left(S(0), R(0), x_{1}(0), x_{2}(0), x_{3}(0), x_{4}(0), x_{5}(0)\right)=(0.3,0.1,0.01,0.03,0.02,0.075$, $0.1)$. The top curve depicts $x_{5}(t)$, and the bottom one depicts $x_{4}(t)$. Clearly, both $x_{4}(t)$ and $x_{5}(t)$ approach positive constants. 


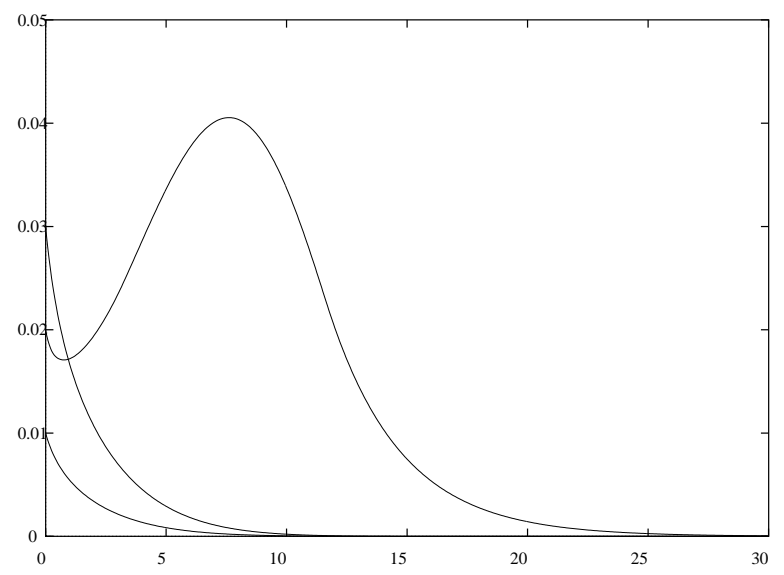

Figure 12: $\left(S(0), R(0), x_{1}(0), x_{2}(0), x_{3}(0), x_{4}(0), x_{5}(0)\right)=(0.3,0.1,0.01,0.03,0.02,0.075$, $0.1)$. The top curve depicts $x_{3}(t)$, the middle curve depicts $x_{2}(t)$, and the the bottom one depicts $x_{1}(t)$. Clearly, all $x_{1}(t), x_{2}(t)$, and $x_{3}(t)$ approach zero. 